\title{
One-Two Punch Therapy for the Treatment of T-Cell Malignancies Involving p53-Dependent Cellular Senescence
}

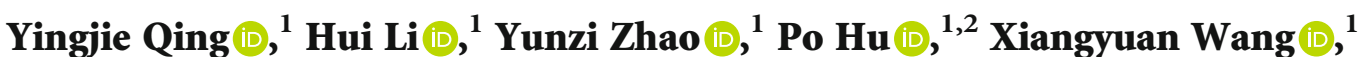 \\ Xiaoxuan Yu $\mathbb{D}^{3}{ }^{3}$ Mengyuan Zhu $\mathbb{D}^{1},{ }^{1}$ Hongzheng Wang $\mathbb{D}^{1},{ }^{1}$ Zhanyu Wang ${ }^{\mathbb{D}},{ }^{1}$ \\ Qinglong Guo $\mathbb{D}^{1}{ }^{1}$ and Hui Hui ${ }^{1}{ }^{1}$ \\ ${ }^{1}$ State Key Laboratory of Natural Medicines, Jiangsu Key Laboratory of Carcinogenesis and Intervention, \\ China Pharmaceutical University, 24 Tongjiaxiang, Nanjing 210009, China \\ ${ }^{2}$ School of Pharmacy, Nanjing University of Chinese Medicine, Nanjing, Jiangsu, 210023, China \\ ${ }^{3}$ Department of Pharmacology, School of Medicine \& Holistic Integrative Medicine, Nanjing University of Chinese Medicine, \\ Nanjing 210023, China
}

Correspondence should be addressed to Qinglong Guo; anticancer_drug@163.com and Hui Hui; moyehh@163.com

Yingjie Qing and Hui Li contributed equally to this work.

Received 13 February 2021; Accepted 27 June 2021; Published 22 September 2021

Academic Editor: Felipe L. de Oliveira

Copyright (C) 2021 Yingjie Qing et al. This is an open access article distributed under the Creative Commons Attribution License, which permits unrestricted use, distribution, and reproduction in any medium, provided the original work is properly cited.

T-cell malignancies are still difficult to treat due to a paucity of plans that target critical dependencies. Drug-induced cellular senescence provides a permanent cell cycle arrest during tumorigenesis and cancer development, particularly when combined with senolytics to promote apoptosis of senescent cells, which is an innovation for cancer therapy. Here, our research found that wogonin, a well-known natural flavonoid compound, not only had a potential to inhibit cell growth and proliferation but also induced cellular senescence in T-cell malignancies with nonlethal concentration. Transcription activity of senescencesuppression human telomerase reverse transcriptase (hTERT) and oncogenic C-MYC was suppressed in wogonin-induced senescent cells, resulting in the inhibition of telomerase activity. We also substantiated the occurrence of DNA damage during the wogonin-induced aging process. Results showed that wogonin increased the activity of senescence-associated $\beta$ galactosidase (SA- $\beta$-Gal) and activated the DNA damage response pathway mediated by $\mathrm{p} 53$. In addition, we found the upregulated expression of BCL-2 in senescent T-cell malignancies because of the antiapoptotic properties of senescent cells. Following up this result, we identified a BCL-2 inhibitor Navitoclax (ABT-263), which was highly effective in decreasing cell viability and inducing apoptotic cell death in wogonin-induced senescent cells. Thus, the "one-two punch" approach increased the sensibility of T-cell malignancies with low expression of BCL-2 to Navitoclax. In conclusion, our research revealed that wogonin possesses potential antitumor effects based on senescence induction, offering a better insight into the development of novel therapeutic methods for T-cell malignancies.

\section{Introduction}

T-cell malignancies are a highly heterogeneous group of disorders $[1,2]$, which have been widely acknowledged to be typically aggressive and resistant to chemotherapy and have poor prognosis [3-5]. T-cell malignancies commonly consist of two categories, T-cell acute lymphoblastic leukemia (T-ALL) and T-cell non-Hodgkin lymphoma (T-NHL), both of which have similar clinical therapies and poor prog- nosis [6]. Due to the varied morphology of subtypes and being highly heterogeneous, the diagnosis and treatment of this disease have become a great challenge [7]. Although the standard first-line treatment of $\mathrm{T}$-cell malignancies is a CHOP regimen (cyclophosphamide, doxorubicin, vincristine, and prednisone), which is still the representative chemotherapy [8], most patients still have a poor prognosis with an ORR of $65.8 \%$ because of rapid relapse [8]. Relapse and cytotoxicity always become an unavoidable problem for 
several patients. And just a few of patients who achieve remission are suitable for hematopoietic stem cell transplantation $[9,10]$. Thus, it is urgent to find more effective therapy options for patients who fail to respond to chemotherapy or relapse after chemotherapy.

Cellular senescence is a complex stress response accompanied by permanent cell cycle arrest and inhibition of proliferation, which is induced by DNA damage, telomere shortening, ionizing radiation, or oncogene $[11,12]$. The typical characteristics of senescent cells are large and flat morphology and positive expression of senescenceassociated $\beta$-galactosidase. Moreover, senescence-associated heterochromatin foci (SAHF) are also a biomarker of senescent cells, which are formed by nuclei as the heterochromatin structures [13]. In hematologic malignancies, cells abnormally overexpress human telomerase reverse transcriptase and decrease the expression of senescence-promoting genes, which contribute to immortalization of cancer cells [14]. Previous reports have shown that $h T E R T$ also exerts antisenescent effect through telomerase- and telomere lengthindependent mechanisms [15]. As a novel therapeutic concept, the use of drugs which can accumulate DNA damage and trigger cellular senescence in cancer cells can provide a new insight into treating hematologic malignancies. However, induced cellular senescence alone cannot achieve the ideal therapeutic effect. Many chemotherapy drugs can induce cellular senescence and alert the tumor microenvironment, which is a double-edged sword for cancer therapy [11, 16]. Although senescent cells induced by agents had vulnerabilities such as persistent growth inhibition, persisting existence of senescent cells can promote cancer progression and invasion in B-cell lymphoma and T-cell acute lymphoblastic leukemia [17]. Thus, exploiting vulnerabilities and removing senescent cells are important for the second step of senescence-focused cancer therapies [18-20]. As the characteristic of resistance to apoptosis in senescent cells, senolytics are identified to eliminate aging cells including quercetin/dasatinib, pan-BCL inhibitors through the inhibition of the BCL-2 family of proteins (BCL-2, BCL-xL, and BCL-w) $[21,22]$. Therefore, a one-two punch cancer therapy may be regarded as a potentially therapeutic strategy [20].

Nowadays, DNA-damaging drugs are widely used in cancer therapy, which not only induce cell death but also activate cellular senescence [23]. DNA damage response (DDR) plays a core role in senescence. The biomarker of DDR is the activation of H2A.X, which depends on the phosphorylation at Ser139 $(\gamma-\mathrm{H} 2 \mathrm{AX})$. As a main factor of DDR, p53 acts as a protagonist during the process of aging and tumor suppression [24]. A previous study reported that in adult acute lymphoblastic leukemia, the alterations of TP53, the most important tumor suppressor gene, including deletions, sequence mutations, and defect in its expression, result in an adverse prognosis [25]. As the activation of DNA damage, p53 is activated through phosphorylation on serine-15 and serine-20 by ataxia-telangiectasia mutated (ATM) or ataxiatelangiectasia Rad3-related (ATR) and the checkpoint kinase 2 (CHK2), respectively [26, 27]. And then, the interaction of MDM2 and p53 is impaired, promoting the accumulation and stabilization of $\mathrm{p} 53$ in response to DNA damage activa- tion [28]. After activation of p53, the downstream effectors like p21 regulate different cell responses such as cell cycle arrest and senescence.

Wogonin (5,7-dihydroxy-8-methoxy-2-phenyl-4h-1benzopyran-4-one) is a type of flavonoid extracted from $S$. baicalensis Georgi, which has prominent biological activities including anti-inflammatory, antiviral, and anticancer activities $[29,30]$. However, cytostatic concentrations of wogonin have other characteristics such as inducing cellular senescence, which can combine with senolytics as a new therapeutic regimen. Here, we first investigated wogonininduced cellular senescence and found the suppression of telomerase activity in wogonin-treated senescent cells. Moreover, DNA damage response mediated by p53 was involved in cellular senescence induced by wogonin. Finally, we also clarified that the combination sequential treatment of wogonin and Navitoclax, a BCL-2 inhibitor, could obtain better effect and become a novel strategy in T-cell malignancies.

\section{Materials and Methods}

2.1. Compounds and Reagents. Wogonin (purity $\geq 99 \%$ ) is isolated from $S$. baicalensis Georgi according to previously reported protocols [31]. Wogonin was dissolved in dimethyl sulfoxide (DMSO) as a stock solution $(100 \mathrm{mM})$ and stored at $-80^{\circ} \mathrm{C}$. The solution was freshly diluted with basal medium to designated concentrations, and the final concentration of DMSO will not be over $0.1 \%$. Cells treated with the highest concentration of DMSO were used as the control in corresponding experiments. Navitoclax (CSN12932) was purchased from CSNpharm (Chicago, USA). Navitoclax powder was dissolved with DMSO to $10 \mathrm{mM}$ and stored at $-20^{\circ} \mathrm{C}$. Primary antibodies against $\beta$-actin, GAPDH, trimethyl-histone H3-K9, BCL-2, hTERT, c-Myc, caspase-3, active caspase-3, p27, Bax, CHK2, cyclin D1, cyclin E1, CDK4, CDK6, HRP goat anti-mouse IgG $(\mathrm{H}+\mathrm{L})$, and HRP goat anti-rabbit $\operatorname{IgG}(\mathrm{H}+\mathrm{L})$ were obtained from ABclonal Technology (Wuhan, China). Phospho-CHK2 (T68), phospho-p53 (Ser15), p21, and phospho-histone H2A.X ( $\gamma$ H2A.X) were obtained from Cell Signaling Technology (Danvers, MA, USA). p53, p16, BCL-xL, Bim, and PARP-1 were obtained from Proteintech (CA, USA).

2.2. Cell Culture. The human T-ALL cell line (Jurkat cells), human T-cell lymphoma cell lines (Hut-102 and Hut-78 cells), and HEK293T cells were purchased from the Cell Bank of Shanghai Institute of Biochemistry \& Cell Biology. All the cells were expanded and stored in liquid nitrogen upon receipt, and each aliquot was passaged for fewer than 25-30 times in our laboratory. Jurkat, Hut-102, and Hut-78 cells were cultured in RPMI-1640 medium (Gibco, Carlsbad, USA). HEK293T cells were cultured in DMEM medium (Gibco, Carlsbad, USA). Medium was supplemented with $10 \%$ FBS (Thermo Fisher Scientific, Waltham, MA), $100 \mathrm{U} / \mathrm{mL}$ of penicillin, and $100 \mathrm{U} / \mathrm{mL}$ of streptomycin in a humidified environment (Thermo Fisher Scientific) with $5 \% \mathrm{CO}_{2}$ at $37^{\circ} \mathrm{C}$. 
2.3. Cell Viability Assay. Cell proliferation of cells was detected using Cell Counting Kit-8 (KeyGen Biotech, Nanjing, China) according to the manufacturer's instruction. Briefly, cells $\left(1 \times 10^{4}\right.$ cells/well $)$ were seeded into a 96-well plate in $100 \mu \mathrm{L}$ medium and treated with wogonin at different concentrations for $24 \mathrm{~h}$. Each group consisted of six parallel wells. Then, $10 \mu \mathrm{L}$ of CCK8 solution was added to each well, and cells were incubated at $37^{\circ} \mathrm{C}$ for $1-4 \mathrm{~h}$. The absorbance was measured at $450 \mathrm{~nm}$ using an Automated Microplate Reader ELx800 (BioTek). The cell survival rate (\%) was calculated using the following equation: survival rate $(\%)=\left(\mathrm{OD}_{\text {treated }}-\mathrm{OD}_{\text {blank }}\right) /\left(\mathrm{OD}_{\text {standard }}-\mathrm{OD}_{\text {blank }}\right) \times 100$ $\%$.Inhibition rate $(\%)=100 \%$ - survival rate $(\%)$.

2.4. Quantitative Real-Time RT-qPCR. RT-qPCR was performed according to the manufacturer's instructions [32].

The primer sequences were as follows:

GAPDH

Forward 5 ' -GCAGGGGGGAGCCAAAAGGG-3'

Reverse $5^{\prime}$-TGCCAGCCCCAGCGTCAAAG-3'

P16

Forward $5^{\prime}$-GATCCAGGTGGGTAGAAGGTC-3'

Reverse $5^{\prime}$-CCCCTGCAAACTTCGTCCT-3'

P21

Forward $5^{\prime}$-TGTCCGTCAGAACCCATGC-3'

Reverse $5^{\prime}$-AAAGTCGAAGTTCCATCGCTC-3'

P27

Forward 5 ' -AACGTGCGAGTGTCTAACGG-3'

Reverse $5^{\prime}$-CCCTCTAGGGGTTTGTGATTCT-3' $C-M Y C$

Forward 5' -GGCTCCTGGCAAAAGGTCA-3'

Reverse $5^{\prime}$-CTGCGTAGTTGTGCTGATGT-3' hTERT

Forward 5' -TATGTCACGGAGACCACGTT-3 ${ }^{\prime}$

Reverse $5^{\prime}$-GTGCTGTCTGATTCCAATGC-3'

2.5. Flow Cytometric Analysis of Apoptosis. Apoptosis was evaluated using an Annexin V-FITC/PI Apoptosis Detection Kit (KeyGen Biotech, Nanjing, China) according to the manufacturer's instruction. Cellular fluorescence was performed by flow cytometric analysis using a FACSCalibur flow cytometer (Becton Dickinson Biosciences, San Jose, CA, USA). Our data was analyzed by using FlowJo software.

2.6. Cell Cycle Analysis. Cells were collected and washed with PBS and then fixed with $70 \%$ ethanol for $2 \mathrm{~h}$ at $4^{\circ} \mathrm{C}$. After fixation, the cells were washed with PBS buffer and stained with PI for $30 \mathrm{~min}$. The fluorescence was detected by flow cytometry. The cell cycle was analyzed and quantified using a BD FACSCalibur flow cytometer (Becton Dickinson, San Jose, CA). The percentage of cells in G0/G1, S, and G2/M phases of the cell cycle was determined by the PI fluorescence signal peak versus the integral which was analyzed by using ModFit LT 4.0 software (Becton Dickinson, San Jose, CA, USA).

2.7. CFSE Assay. CFSE-labeled cells (CellTrace CFSE Proliferation Kit, Invitrogen) were cultured with wogonin for the indicated time, cells were harvested, and cell proliferation capacity was determined by using a flow cytometer.

2.8. Cell Staining of Senescence-Associated $\beta$-Galactosidase (SA- $\beta$-Gal). SA- $\beta$-Gal activity was measured by using a $\beta$ Galactosidase Staining Kit (Yeasen, Shanghai, China) according to the standard protocol. The stained cells were observed and photographed under the microscope (Carl Zeiss, Germany). Cells were considered positive when the cytoplasm was stained with SA- $\beta$-Gal. Total numbers of cells and SA- $\beta$-Gal-positive cells were counted for five fields per well. The ratios of SA- $\beta$-Gal-positive cells were calculated compared to the total cell numbers per area.

2.9. Flow Cytometric Detection of $S A-\beta-G a l$. For flow cytometry-based detection of senescence, the fluorogenic substrate 5-dodecanoylaminofluorescein di-beta-d-galactopyranoside $\left(\mathrm{C}_{12} \mathrm{FDG}\right)$ was used as described previously [33]. According to the manufacturer's instructions, cells were incubated with $33 \mu \mathrm{M} \mathrm{C}{ }_{12}$ FDG (ImaGene Green $\mathrm{C}_{12}$ FDG lacZ Gene Expression Kit) at $37^{\circ} \mathrm{C}$ for $30 \mathrm{~min}$. Then, cells were washed with PBS and measured immediately with a FACSCalibur flow cytometer (Becton Dickinson Biosciences, San Jose, CA, USA). Data were analyzed using FlowJo software. SA- $\beta$-Gal activity was estimated using the relative mean fluorescence intensity of the cell population.

2.10. Western Blotting Analysis. Whole cell proteins were collected after the treatment with wogonin and were extracted by using RIPA buffer containing protease/phosphatase inhibitors on ice for $50 \mathrm{~min}$. Then, cell lysates were clarified by centrifugation at 14,000 rpm (5430R; Eppendorf, Hamburg, Germany) for $25 \mathrm{~min}$ at $4^{\circ} \mathrm{C}$. Concentrations of proteins were measured using the BCA Protein Assay Kit (Thermo Fisher Scientific, USA). Protein extracts were equally loaded on $8 \%-12 \%$ SDS-polyacrylamide gels and then transferred onto the PVDF membranes (Millipore, Boston, MA, USA). Membranes were blocked with $3 \%$ BSA in PBS at room temperature for $1 \mathrm{~h}$ and then incubated with primary antibodies overnight at $4^{\circ} \mathrm{C}$. After that, the membrane was incubated with an HRP goat anti-rabbit IgG $(\mathrm{H}+\mathrm{L})$ or HRP goat antimouse IgG $(\mathrm{H}+\mathrm{L})$ secondary antibody for $1 \mathrm{~h}$ at room temperature. Chemiluminescence detection was performed by using ECL reagents (Thermo Fisher Scientific, USA) upon using the AI600 imaging system (GE Healthcare, Pittsburgh, PA, USA).

2.11. Immunofluorescence. Cells were collected and seeded onto glass coverslips. Then, the cells were fixed in the icecold methanol for $10 \mathrm{~min}$ and incubated with $0.15 \%(v / v)$ Triton X-100 for $20 \mathrm{~min}$. After the cells were blocked with $3 \%$ BSA at room temperature for $1 \mathrm{~h}$, the cells were incubated with primary antibodies overnight at $4^{\circ} \mathrm{C}$. Following this, the cells were stained with an Alexa Fluor 488-conjugated goat anti-rabbit IgG secondary antibody (Becton Dickinson) for $1 \mathrm{~h}$ and DAPI (Beyotime Biotechnology, Shanghai, China) for $5 \mathrm{~min}$. Cells were observed under a confocal laser scanning microscope (FV1000; Olympus, Tokyo, Japan).

2.12. RNA Interference. For RNA interference by lentiviral vectors, P53 shRNA constructs and a negative control 
construct created in the same vector system (PLV3ltr-puroU6) were purchased from Corues Biotechnology. A P53 shRNA construct and a negative control were transfected into HEK293T cells together with the Lentiviral Mix and HG Transgene ${ }^{\mathrm{TM}}$ Reagent according to the manufacturer's instruction of the Lentiviral Packaging Kit (Yeasen, $41102 \mathrm{ES} 20)$ for $48 \mathrm{~h}$. After this, the virus supernatant was harvested. Then, the virus supernatant infected target cells. $2 \mu \mathrm{g} / \mathrm{mL}$ puromycin was added to screen for expressing shRNA construct cells.

2.13. Statistical Analysis. All results were expressed as the mean \pm standard error of the mean (SEM). The data shown were obtained from triplicate independent parallel experiments. Statistical analyses were performed by using one-way analysis of variance (ANOVA), post hoc intergroup comparisons, and Tukey's test. Two-tailed Student's $t$-test was used to determine differences between groups. ${ }^{*} p<0.05$ was considered statistically significant, ${ }^{* *} p<0.01$ very significant, and ${ }^{* * *} p<0.001$ highly significant. Statistical analyses were performed using GraphPad Prism 6.0 Software (GraphPad, San Diego, CA).

\section{Results}

3.1. Wogonin Induced Growth Inhibition but Not Apoptosis in Hut-102 and Jurkat Cells. In order to investigate the growth suppression effects of wogonin on T-cell malignancy cells, the growth inhibition rates of cells were detected by the CCK8 assay firstly. We performed our studies using T-NHL cell lines (Hut-102 and Hut-78) and the T-ALL cell line (Jurkat). Cells were treated with various concentrations of wogonin $(0-256 \mu \mathrm{M})$ for $24 \mathrm{~h}$. As shown in Figure 1(a), wogonin inhibited cell proliferation in a concentrationdependent manner. To investigate whether low concentration of wogonin still has proliferation inhibition on T-cell malignancies for a long-time exposure, we chose $20 \mu \mathrm{M}$ as the treatment concentration. $20 \mu \mathrm{M}$ wogonin inhibited cell proliferation in T-cell malignancies notably, as the decreased fluorescence intensity in CFSE-labeled cells was prevented by wogonin (Figures 1(b) and 1(c)). However, $20 \mu \mathrm{M}$ wogonin did not induce significant apoptosis in Hut-102 and Jurkat cells after treatment for $1,3,5$, and $7 \mathrm{~d}$, but in Hut-78 cells, apoptosis rates showed a slight increase (Figure 1(d)). It is concluded that wogonin suppressed growth and proliferation of human T-cell malignancy cells.

3.2. Wogonin Induced Cellular Senescence in Hut-102 and Jurkat Cells but Not Hut-78 Cells. A previous study has confirmed that small-molecule compounds can induce the cellular senescence in a few of cases [34]. It is a viable therapeutic option named one-two punch therapy to induce senescence of cancer cells and then remove them [19]. In order to validate the one-two punch therapy for the treatment of T-cell malignancies, firstly we identified whether wogonin could induce cellular senescence in these T-cell malignancy cells. $\mathrm{C}_{12} \mathrm{FDG}$ and senescence-associated $\beta$-galactosidase staining test were adopted to assess the cellular senescence in Hut102, Jurkat, and Hut-78 cells treated with $20 \mu \mathrm{M}$ wogonin for $5 \mathrm{~d}$. Higher SA- $\beta$-Gal activity measured by $\mathrm{C}_{12}$ FDG using flow cytometry was observed in wogonin-treated Hut-102 and Jurkat cells (Figures 2(a) and 2(b)). Moreover, more positive $\beta$-galactosidase-stained cells were found in wogonin-treated Hut-102 and Jurkat cells compared with control cells (Figures 2(c) and 2(d)). Morphologically, the senescent cells were enlarged and flattened compared with control cells (Figures 2(c) and 2(d)). Interestingly, cellular senescence was not observed in Hut-78 cells after wogonin treatment for $5 \mathrm{~d}$ (Figures $2(\mathrm{a})-2(\mathrm{~d})$ ). Overall, these results showed that low concentration of wogonin $(20 \mu \mathrm{M})$ could induce cellular senescence in Hut-102 and Jurkat cells.

3.3. Wogonin-Induced Cell Cycle Arrest Was Accompanied by Cellular Senescence. Cell cycle blockage is a well-known feature of cellular senescence, which contributes to permanent growth arrest in senescent cells $[35,36]$. Thus, the effects of wogonin on cell cycle progression were measured in Hut102 and Jurkat cells treated with $20 \mu \mathrm{M}$ wogonin for $5 \mathrm{~d}$. Results showed that the proportion of cells increased in the $G_{1}$ phase and decreased in the $S$ phase compared to the untreated cells (Figures 3(a) and 3(b)). The CDK4/6-cyclin D1 complex plays a key role in promoting transition of the $\mathrm{G}_{1}$ to $S$ phrase, and the CDK2-cyclin E1 complex, the downstream of the CDK4/6-cyclin D1 complex, promotes cells to enter the $S$ phrase through the restriction point [37]. Western blots indicated that wogonin decreased the expression of cyclin D1, CDK4, and CDK6, while the cyclin E1 expression was stable (Figures 3(c) and 3(d)). Furthermore, the increased expression of p16,p21, and p27 may contribute to cell cycle blockage in senescent cells [38,39]. RT-PCR results showed that wogonin increased the mRNA level of $P 21$ by over 20-fold in senescent Hut-102 and Jurkat cells, while the mRNA level of $P 27$ did not change in those two cell lines (Figure 3(e)). The mRNA level of P16 was slightly increased in Jurkat cells but not in Hut-102 cells (Figure 3(e)). All these data demonstrated that wogonin arrested the cell cycle at the checkpoint of $G_{0} / G_{1}$, which was correlated with cellular senescence in T-cell malignancy cell lines including Hut102 and Jurkat.

3.4. Wogonin Suppressed Telomerase Activity by Inhibition of hTERT and c-Myc in Wogonin-Induced Senescent Cells. Telomere length affects cell life, and when the telomere shortens to a certain extent, cell senescence occurs [40]. Previous studies have shown that telomerase activity affects telomere length [41]. As a main component of telomerase, human telomerase reverse transcriptase is overexpressed in many malignant tumors including hematologic malignancies [42, 43]. c-Myc, a transcription factor, binds to the promoter region of $h T E R T$ and drives overexpression of $h T E R T$ in cancer cells, resulting in the activation of telomerase [44]. In order to verify whether wogonin induced cellular senescence involved in inhibiting telomerase activity, we first detect the mRNA levels of $C-M Y C$ and $h T E R T$. Results showed that the mRNA expression of $C-M Y C$ and $h T E R T$ were decreased in Hut-102 and Jurkat cells after treatment with $20 \mu \mathrm{M}$ wogonin for $5 \mathrm{~d}$ (Figure 4(a)). By the way, we also investigate the protein expression of c-Myc and hTERT using western blot, 

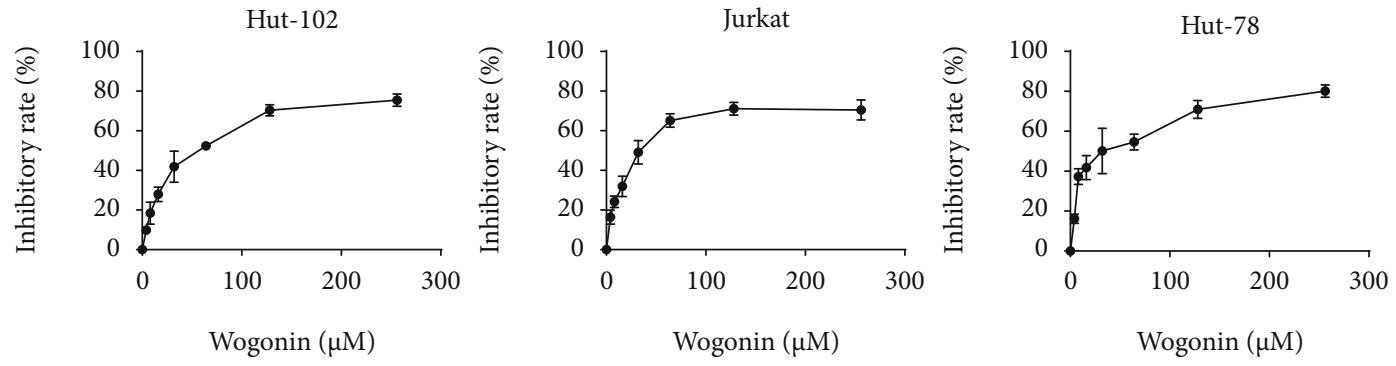

(a)

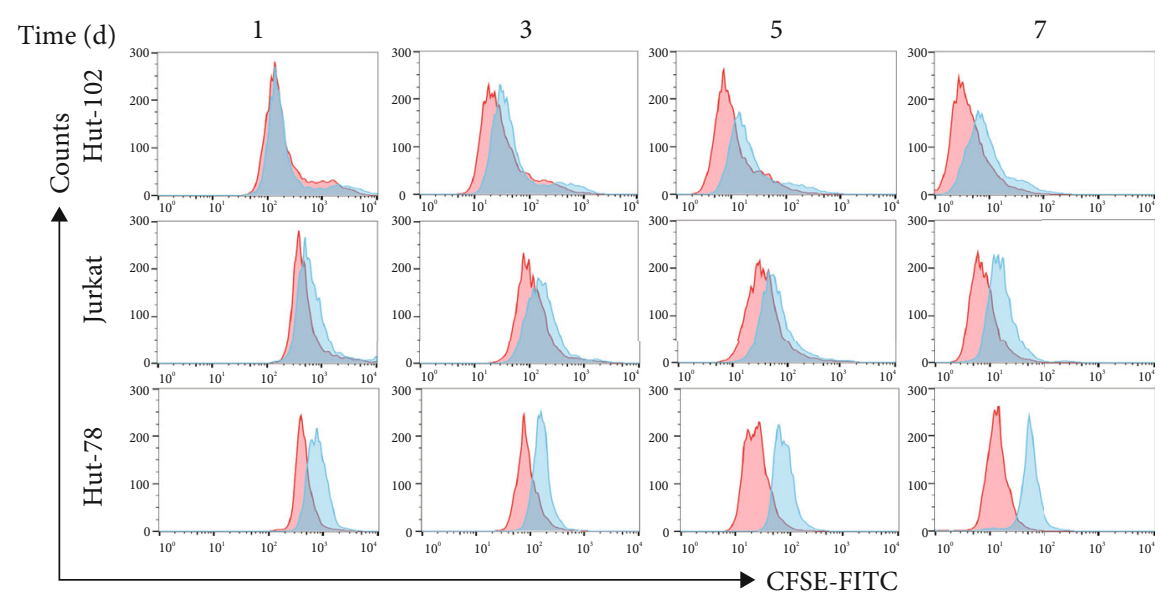

Wogonin $(0 \mu \mathrm{M})$

Wogonin $(20 \mu \mathrm{M})$

(b)

FIgure 1: Continued. 

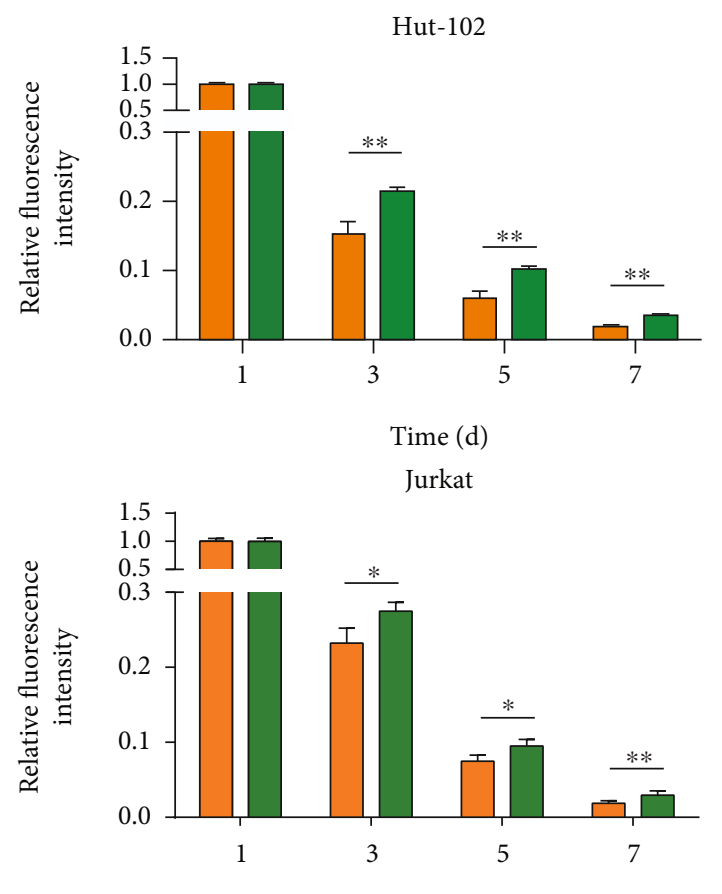

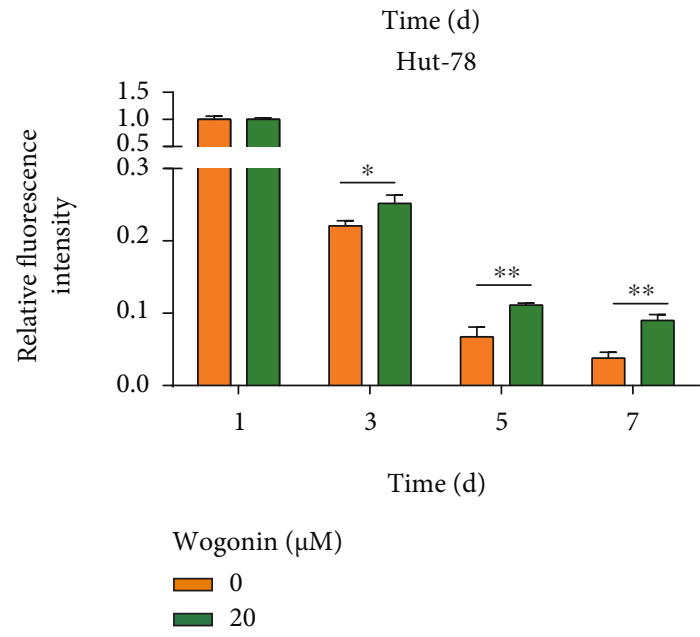

(c)

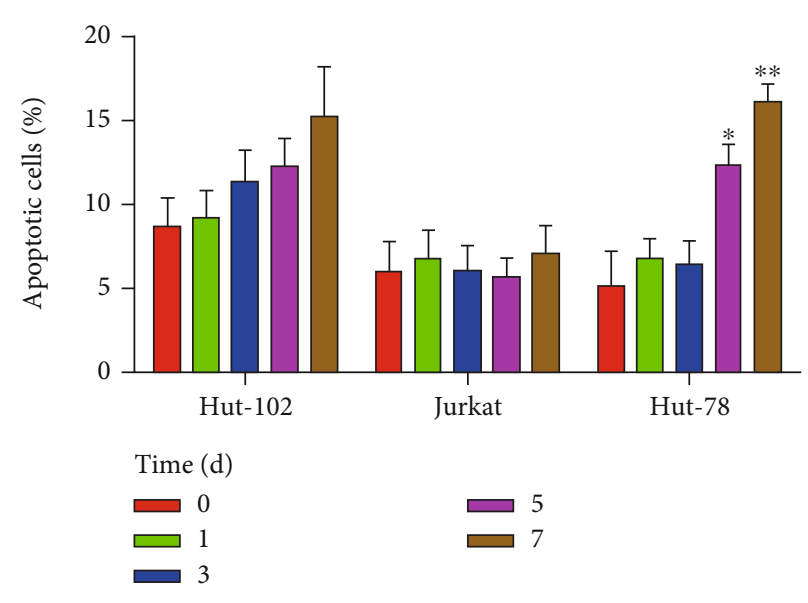

(d)

Figure 1: Wogonin induced growth inhibition but not apoptosis in Hut-102 and Jurkat cells. (a) Hut-102, Jurkat, and Hut-78 cells were exposed to wogonin at indicated concentrations $(0-256 \mu \mathrm{M})$, respectively. The inhibitory rate of cell viability was measured by the CCK8 assay after $24 \mathrm{~h}$. (b) Hut-102, Jurkat, and Hut-78 cells were treated with or without $20 \mu \mathrm{M}$ wogonin; then, the cells were labeled with CFSE for 1, 3, 5, and $7 \mathrm{~d}$, respectively. Results of CFSE expression were analyzed by flow cytometry. (c) Quantification of CFSE expression. Ordinate represents relative changes of fluorescence intensity (GEOmean). Significant difference represents the fluorescent intensity between the control group and wogonin group in the same day. Columns represent the mean from three parallel experiments (mean $\pm \mathrm{SEM}) .{ }^{*} p<0.05,{ }^{* *} p<0.01$, compared with the control group. (d) Hut-102, Jurkat, and Hut-78 cells were treated with $20 \mu \mathrm{M}$ wogonin for the indicated times $(0,1,3,5$, and $7 \mathrm{~d})$. Then, the cell apoptosis rates were analyzed via Annexin V/PI staining by flow cytometry. Columns represent the mean from three parallel experiments (mean $\pm \mathrm{SEM}$ ). ${ }^{*} p<0.05,{ }^{* *} p<0.01$, compared with the control group.

and results showed that wogonin decreased the expression of c-Myc and hTERT in Hut-102 and Jurkat cells after treatment for $5 \mathrm{~d}$ (Figures 4(b) - 4(e)). Thus, we concluded that wogonin could inhibit telomerase activity in senescent cells.

3.5. Wogonin Induced Senescence-Associated Heterochromatin Foci in Senescent Cells. Cell senescence is accompanied by the change of chromosome structure, especially the phenomenon of heterochromatin foci, which is the biological marker of cell senescence $[13,45]$. In order to determine whether wogonin induced SAHF in senescent cells, Hut-102 and Jurkat cells were exposed to $20 \mu \mathrm{M}$ wogonin for $5 \mathrm{~d}$ and formation of DNA agglutination points appeared (Figure 5(a)). The ratios of agglutination point-positive cells were $38.00 \% \pm 5.10 \%$ and $44.22 \pm 11.09 \%$ in wogonin-treated Hut-102 and Jurkat cells, respectively (Figure 5(b)). Next, we verified the molecular marker of SAHF named H3K9me3 by western blots and under a laser scanning confocal microscope. Results of 


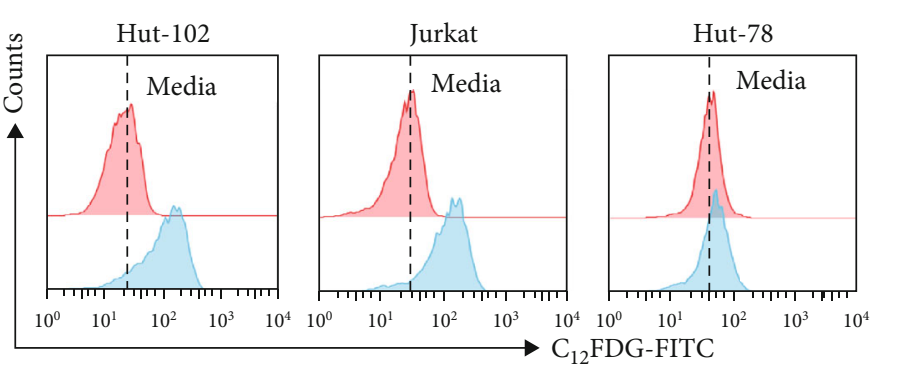

Wogonin $(\mu \mathrm{M})$
$\square 0$
$\square 20$

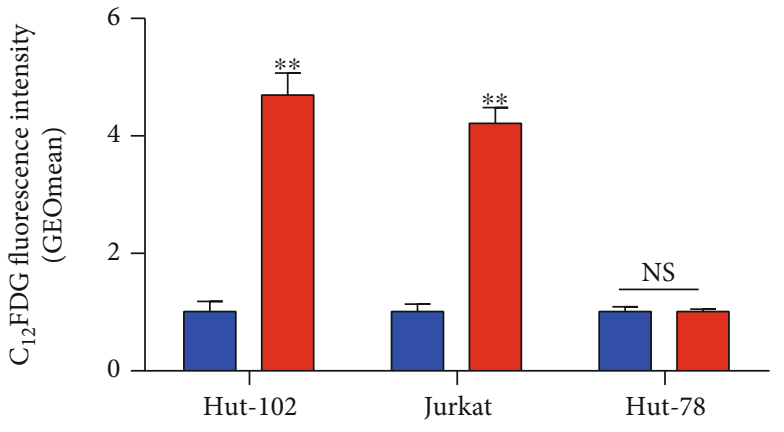

Wogonin $(\mu \mathrm{M})$

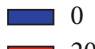

$\square 20$ (a)

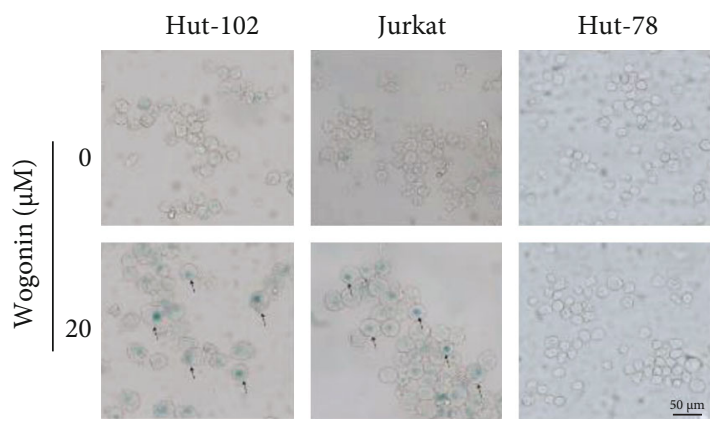

(c)

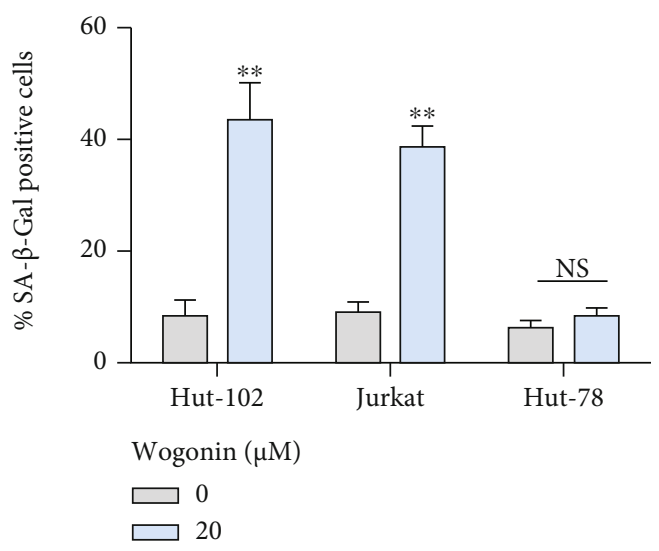

(d)

FIGURE 2: Wogonin induced cellular senescence in Hut-102 and Jurkat cells but not Hut-78 cells. (a) Cells (Hut-102, Jurkat, and Hut-78 cells) were treated with or without $20 \mu \mathrm{M}$ wogonin for $5 \mathrm{~d}$. SA- $\beta$-Gal activity was measured by $\mathrm{C}_{12}$ FDG fluorogenic substrate of SA- $\beta$-galactosidase using flow cytometry. Representative peak graphs from one of the three independent experiments, illustrating $\mathrm{C}_{12}$ FDG fluorescence. (b) Quantification of SA- $\beta$-Gal activity measured by $\mathrm{C}_{12} \mathrm{FDG}$ fluorescence. The dark blue histogram represents control cells, and the red histogram represents cells that were cultured in the presence of $20 \mu \mathrm{M}$ wogonin. Columns represent the mean from three parallel experiments (mean $\pm \mathrm{SEM}$ ). ${ }^{*} p<0.05,{ }^{* *} p<0.01$, compared with the control group. (c) SA- $\beta$-Gal activity was measured by SA- $\beta$-Gal staining in cells (Hut-102, Jurkat, and Hut-78 cells) treated with or without $20 \mu \mathrm{M}$ wogonin for $5 \mathrm{~d}$. Representative images from one of the three independent experiments. Representative pictures are shown (magnification, $\times 200$ ). (d) Quantification of SA- $\beta$-Gal activity measured by cell staining. The grey histogram represents control cells, and the blue histogram represents cells that were cultured in the presence of $20 \mu \mathrm{M}$ wogonin. Columns represent the mean from three parallel experiments (mean \pm SEM). ${ }^{*} p<0.05,{ }^{* *} p<0.01$, compared with the control group.

western blots showed that the expression of $\mathrm{H} 3 \mathrm{~K} 9 \mathrm{me} 3$ was upregulated after the treatment with wogonin for 3,5 , and $7 \mathrm{~d}$ in Hut-102 cells; meanwhile, H3K9me3 was also upregulated after the treatment with wogonin for 5 and $7 \mathrm{~d}$ in Jurkat cells (Figures 5(c) and 5(d)). Immunofluorescence analysis showed that the fluorescence intensity of $\mathrm{H} 3 \mathrm{~K} 9 \mathrm{me} 3$ increased and colocated with the agglutination point in Hut-102 and Jurkat cells (Figure 5(e)).

3.6. Wogonin Induced Cellular Senescence through p53Mediated DNA Damage Response. Many studies have shown that anticancer agent-induced cellular senescence is mediated by the DNA damage pathway $[24,46]$. To figure out the mechanism of cellular senescence induced by wogonin, we first performed western blot experiments to detect the protein expression of the DNA damage marker protein $\gamma$ H2AX in Hut-102 and Jurkat cells. We found that the $\gamma$ $\mathrm{H} 2 \mathrm{AX}$ expression increased obviously after treatment with $20 \mu \mathrm{M}$ wogonin for $5 \mathrm{~d}$ and $7 \mathrm{~d}$ (Figure 6(a)), while in Hut78 cells, the level of $\gamma$-H2AX was increased slightly with the treatment with $20 \mu \mathrm{M}$ wogonin for $7 \mathrm{~d}$ (Figure S1a). In line with that, results of the immunofluorescence experiment showed that the fluorescence intensity of $\gamma$-H2AX was enhanced and colocalized with the nucleus after treatment with $20 \mu \mathrm{M}$ wogonin in three T-cell malignancies (Figure 6(b), Figure S1b). The enhanced expression of $\gamma$ $\mathrm{H} 2 \mathrm{AX}$ proved the wogonin-induced DNA damage response. We then analyzed the activation level of the $\mathrm{CHK} 2 / \mathrm{p} 53 / \mathrm{p} 21$ axis, which is involved in DNA damage response [47]. Western blot indicated that the level of $\mathrm{p}$ - 

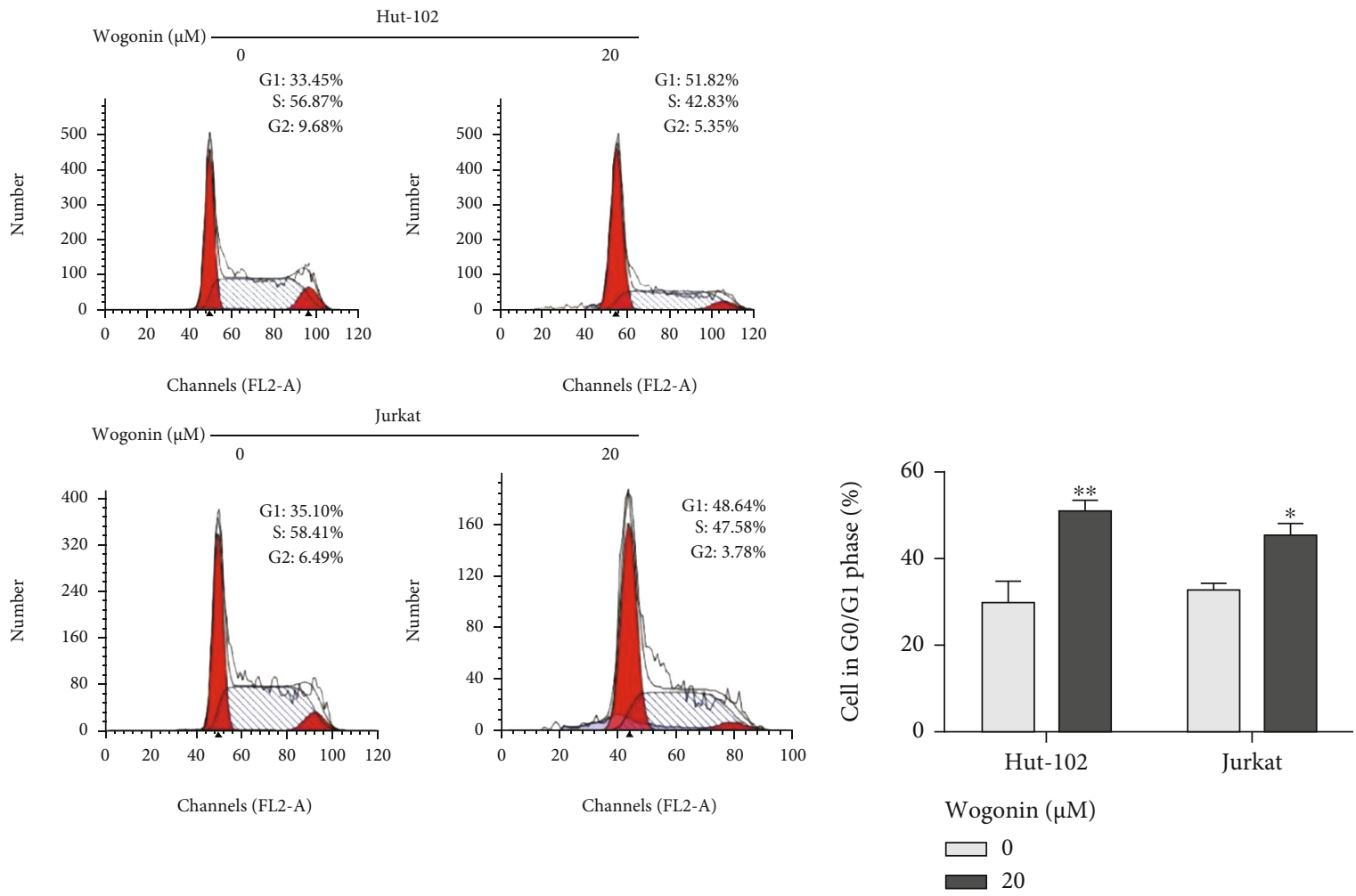

(a)

(b)
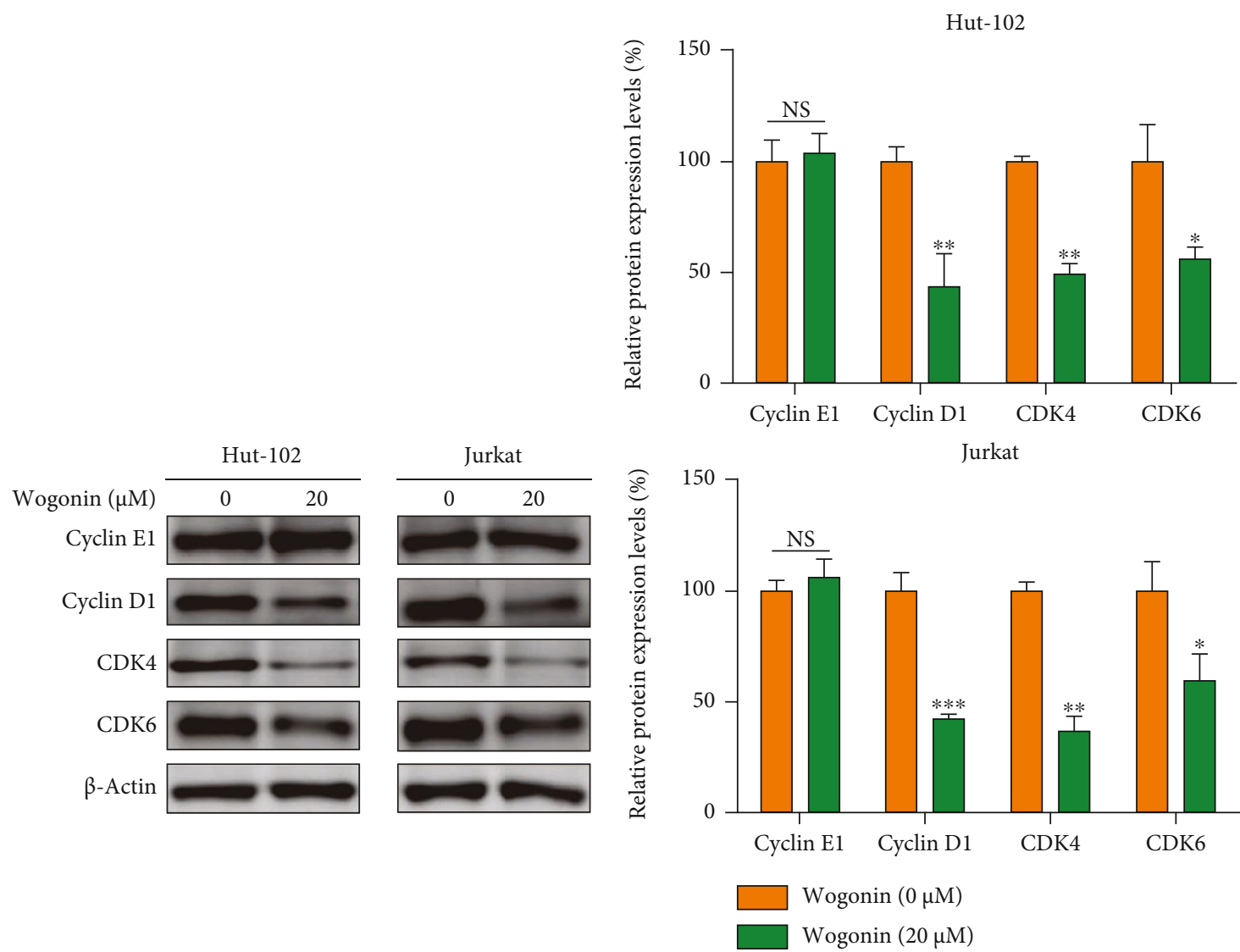

(c)

(d)

Figure 3: Continued. 

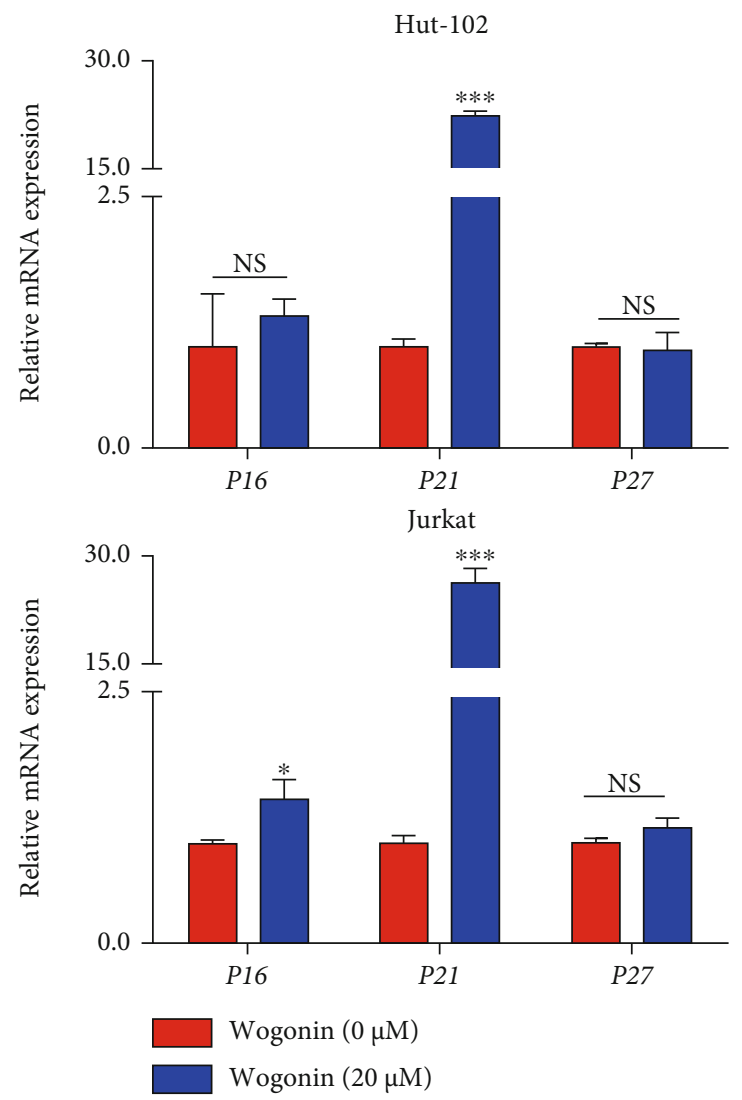

(e)

FIGURE 3: Wogonin-induced cell cycle arrest was accompanied by cellular senescence. (a) Hut-102 and Jurkat cells were treated with or without $20 \mu \mathrm{M}$ wogonin for $5 \mathrm{~d}$. Representative cell cycle was performed by PI staining and analyzed by flow cytometry. (b) The percentages of cells in the $G_{0} / G_{1}$ phases of the cell cycle following $20 \mu \mathrm{M}$ wogonin treatment for $5 \mathrm{~d}$ are shown. Columns represent the mean from three parallel experiments (mean \pm SEM). ${ }^{*} p<0.05,{ }^{* *} p<0.01$, compared with the control group. (c) Hut-102 and Jurkat cells were treated with or without $20 \mu \mathrm{M}$ wogonin for $5 \mathrm{~d}$. Western blot analyses of cell cycle regulatory proteins cyclin E1, cyclin D1, CDK4, and CDK6. $\beta$-Actin was used as loading controls. (d) Relative protein expression levels of cyclin D1, cyclin E1, CDK4, and CDK6 were determined. Columns represent the mean from three parallel experiments (mean $\pm \mathrm{SEM}) .{ }^{*} p<0.05,{ }^{* *} p<0.01$, compared with the control group. (e) The relative mRNA levels of $P 16, P 21$, and $P 27$ in control and wogonin-treated Hut-102 and Jurkat cells. Columns represent the mean from three parallel experiments (mean $\pm \mathrm{SEM}$ ). ${ }^{*} p<0.05,{ }^{* *} p<0.01$, and ${ }^{* * *} p<0.001$, compared with the control group.

CHK2 (Thr68) was gradually upregulated in wogonintreated Hut-102 and Jurkat cells in a time-dependent manner, as well as p-p53 (Ser15) and p21. However, the expression of CHK2, p53 and p16, and p27 was stable in Hut-102 and Jurkat cells after treatment with wogonin (Figures 6(c) and 6(d)). But in Hut-78 cells, a cell line lacking p53 expression resulted from a homozygous point mutation at codon 196 (CGA-TGA) [48] and the level of CHK2 and p-CHK2 (Thr68) was increased after treatment with wogonin for $7 \mathrm{~d}$; meanwhile, p21 was slightly increased and p16 and p27 expression was not changed (Figure S1c, d). However, the change degree of p21 in Hut-78 cells is generally slighter than that in Hut-102 and Jurkat cells (Figure 6(d), Figure S1d).

To further investigate whether wogonin-induced senescence depended on the functionally intact p53, we performed our studies using lentiviral-transfected P53 shRNA and negative control (NC) in wt-p53 Hut-102 cells. Transfected efficiency of P53 shRNA is shown in Figures 6(e) and 6(f). Flow cytometric analysis showed that the activity of SA- $\beta$ -
Gal alleviated in cells transfected with P53 shRNA compared with the negative control group after wogonin treatment (Figures 6(g) and 6(h)). Moreover, western blot showed that wogonin-induced expression of p-p53 (Ser15) and p21 was inhibited by P53 knockdown (Figures 6(e) and 6(f)). As a result, DNA damage response is involved in wogonininduced cellular senescence, and the participation of p53 is critical for the cellular senescence induced by wogonin.

3.7. Wogonin-Induced Cellular Senescence Sensitized Navitoclax to Kill T-Cell malignancy cells. Recent research suggested that the expression of prosurvival networks increased in senescent cells including BCL-2, resulting in resistance to apoptosis [19]. We used senolytic drugs to target senescent cells, which is the second step of one-two punch therapy specific to Achilles' heel of senescent cells [19, 49]. Navitoclax, a senolytic BH3 mimetic drug, can selectively kill senescent cells $[21,50]$. Navitoclax binds to BCL-2, BCL-xL, and BCL-w in vitro, while BCL-2 plays a key role in vivo and high expression of BCL-2 exhibited sensitivity to Navitoclax 

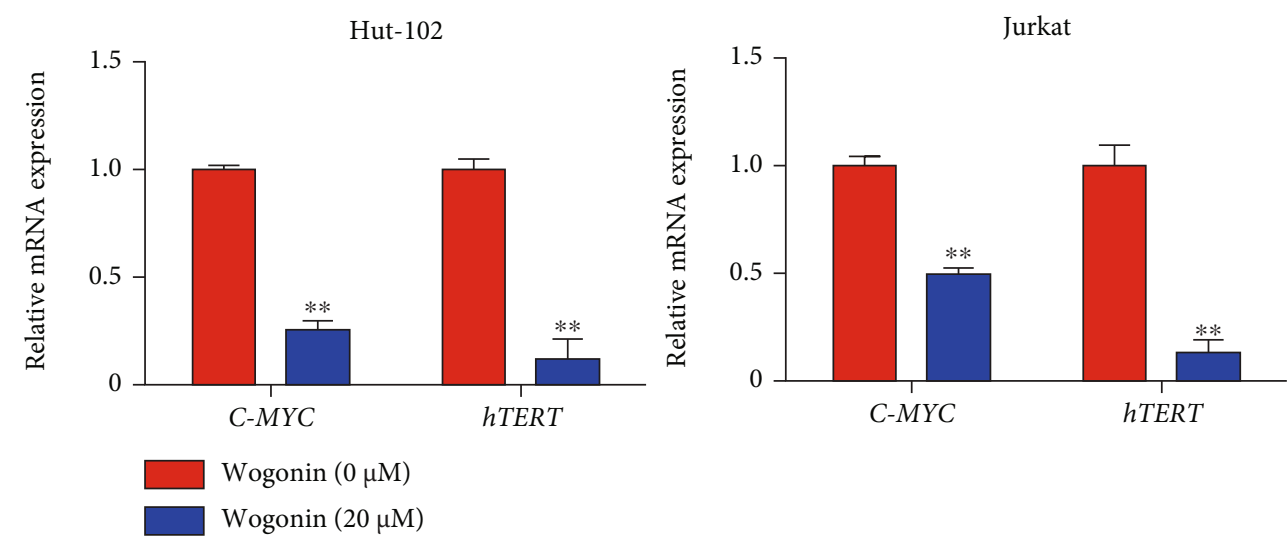

(a)

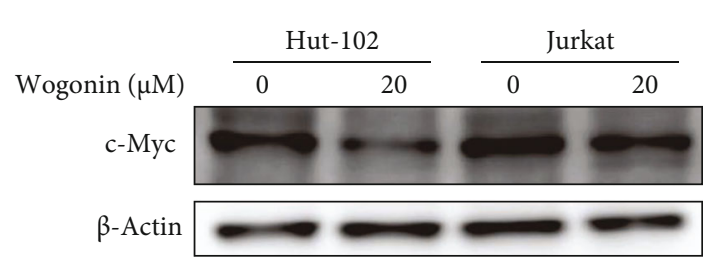

(b)

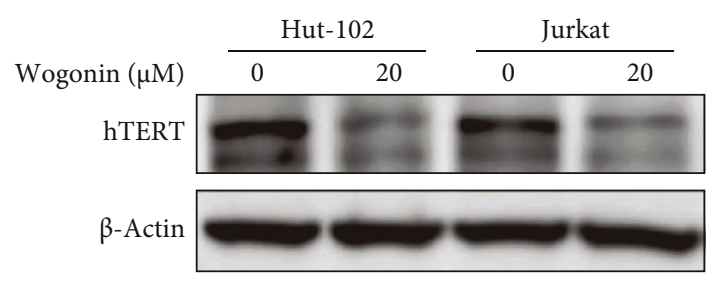

(d)

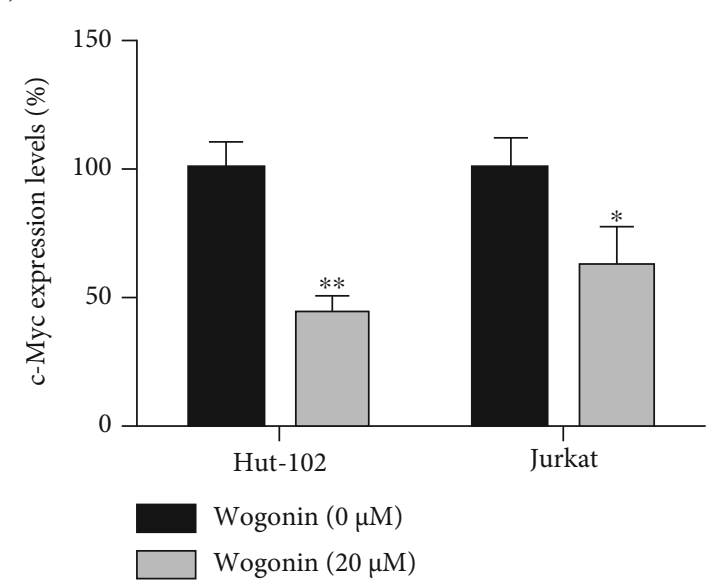

(c)

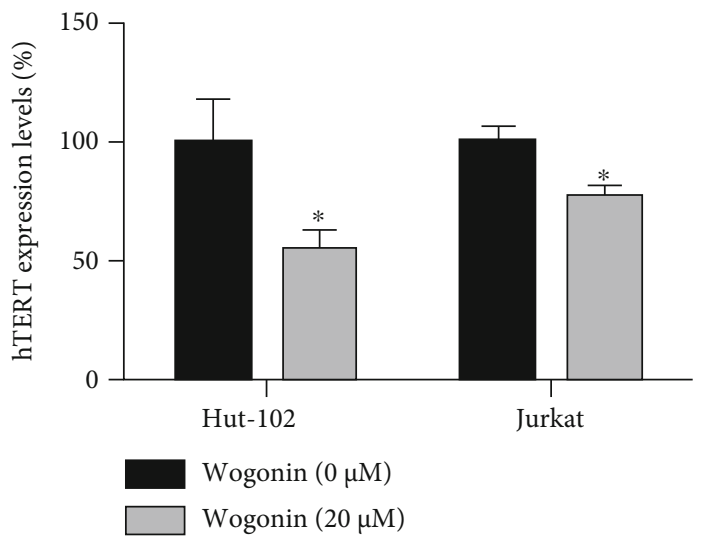

(e)

FIGURE 4: Wogonin suppressed telomerase activity by inhibition of hTERT and c-Myc in wogonin-induced senescent cells. (a) Hut-102 and Jurkat cells were treated with or without $20 \mu \mathrm{M}$ wogonin for $5 \mathrm{~d}$. The relative mRNA level of $h T E R T$ and $C$-MYC was measured by RT-PCR. Columns represent the mean from three parallel experiments (mean $\pm \mathrm{SEM}$ ). ${ }^{*} p<0.05,{ }^{* *} p<0.01$, compared with the control group. (b) Hut102 and Jurkat cells were treated with or without $20 \mu \mathrm{M}$ wogonin for $5 \mathrm{~d}$. The expression of c-Myc protein was performed by western blot. $\beta$ Actin was used as loading controls. (c) Relative protein expression level of c-Myc. Columns represent the mean from three parallel experiments (mean \pm SEM). ${ }^{*} p<0.05,{ }^{* *} p<0.01$, compared with the control group. (d) Hut-102 and Jurkat cells were treated with or without $20 \mu \mathrm{M}$ wogonin for $5 \mathrm{~d}$. The expression of hTERT protein was performed by western blot. $\beta$-Actin was used as loading controls. (e) Relative protein expression level of hTERT. Columns represent the mean from three parallel experiments (mean \pm SEM). ${ }^{*} p<0.05,{ }^{* *} p<0.01$, compared with the control group.

in human non-Hodgkin lymphomas instead of BCL-xL or BCL-w [51]. To investigate whether wogonin-induced senescence promoted the expression of prosurvival protein BCL-2 and BCL-xL, western blot was used and results showed that the expression of BCL-2 was upregulated but the expression of BCL-xL was not changed after treatment with $20 \mu \mathrm{M}$ 


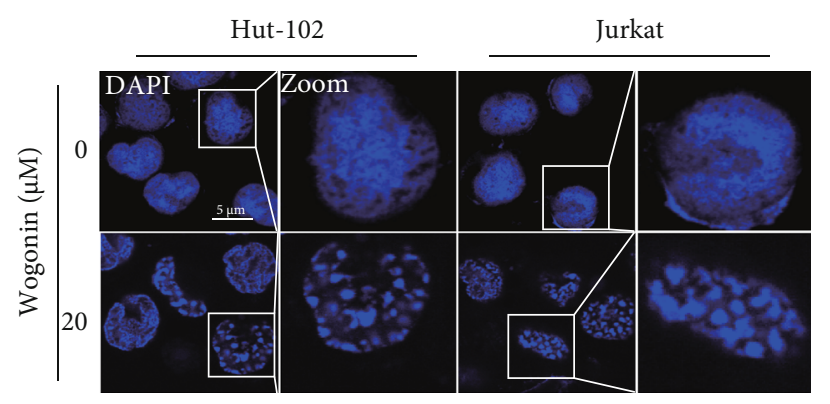

(a)

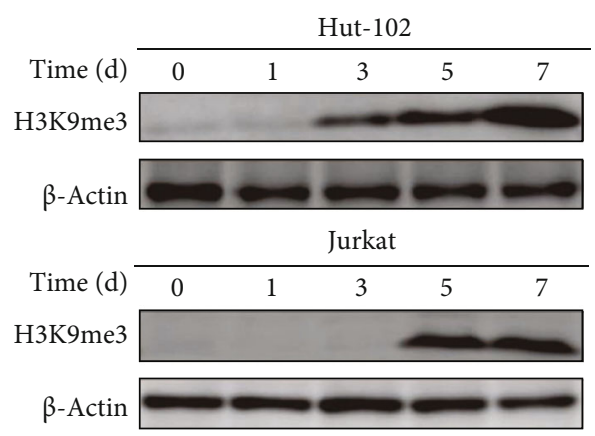

(c)

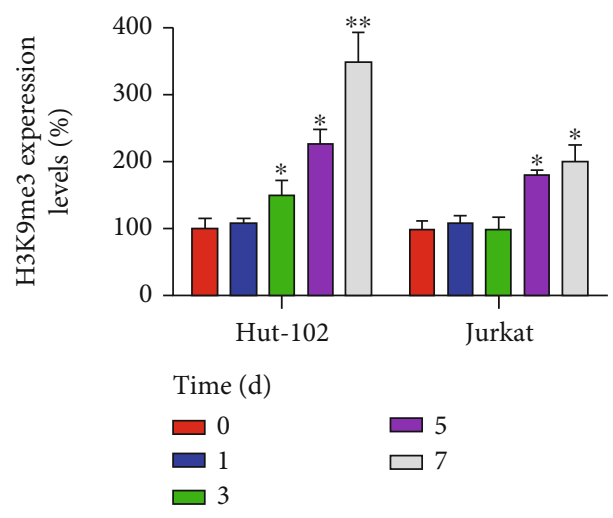

(d)

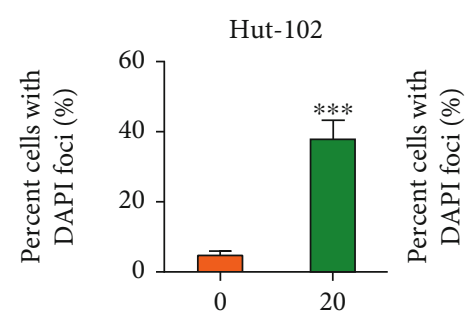

Wogonin $(\mu \mathrm{M})$

(b)
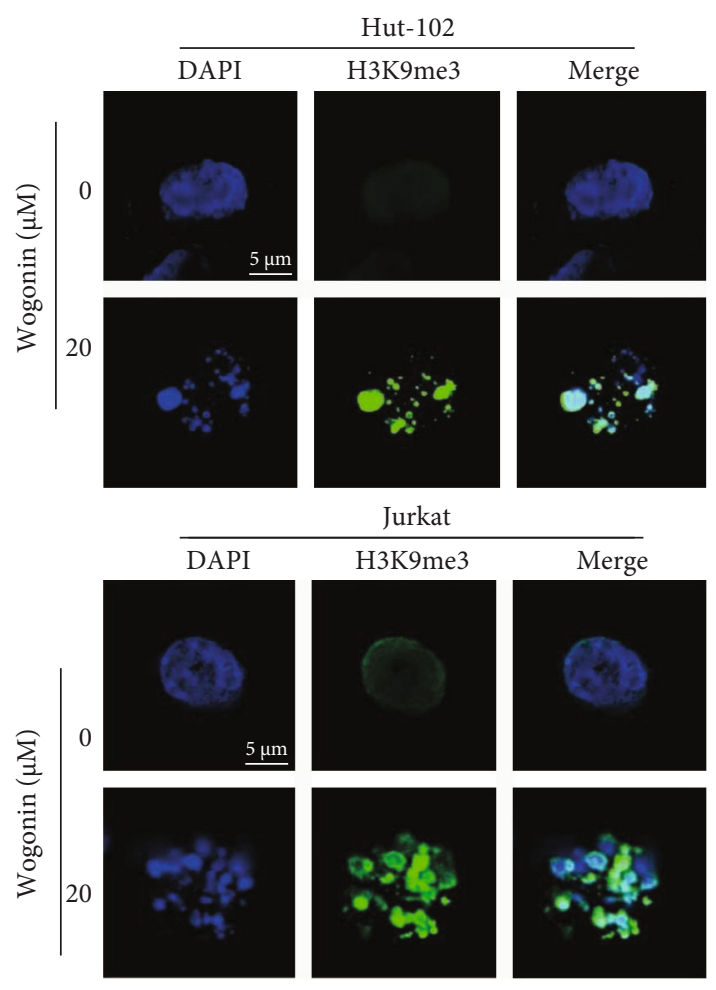

(e)

FIGURE 5: Wogonin induced senescence-associated heterochromatin foci in senescent cells. (a) Hut-102 and Jurkat cells were treated with or without $20 \mu \mathrm{M}$ wogonin for $5 \mathrm{~d}$; then, the cells were fixed and permeabilized, and nuclei were stained with DAPI (blue). SAHF formed in wogonin-treated Hut-102 and Jurkat cells. Representative confocal images for wogonin-induced SAHF formation in cells from confocal laser scanning microscopy are shown (original magnification $\times 1000$; immersion objective $\times 100 / \times 100$ with immersion oil type). Images are representative of three independent experiments. (b) The percentage of positive cells with the DNA agglutination points (DAPI foci) was counted (with 20 cells counted per field). Columns represent the mean from three parallel experiments $($ mean $\pm \operatorname{SEM}) .{ }^{* *} p<0.01,{ }^{* * *} p<$ 0.001, compared with the control group. (c) Hut-102 and Jurkat cells were treated with or without $20 \mu \mathrm{M}$ wogonin for $5 \mathrm{~d}$. The expression of the SAHF marker protein H3K9me3 in control and wogonin-treated Hut-102 and Jurkat cells was performed by western blot. $\beta$-Actin was used as loading controls. (d) Relative protein expression level of $\mathrm{H} 3 \mathrm{~K} 9 \mathrm{me} 3$ was determined. Columns represent the mean from three parallel experiments (mean $\pm \mathrm{SEM}$ ). ${ }^{*} p<0.05,{ }^{* *} p<0.01$, compared with the control group. (e) Hut-102 and Jurkat cells were treated with or without $20 \mu \mathrm{M}$ wogonin for $5 \mathrm{~d}$; then, the cells were fixed, permeabilized, and stained with an antibody against H3K9me3 (green), while nuclei were stained with DAPI (blue). Immunofluorescent images showed the distribution of H3K9me3 in Hut-102 and Jurkat cells (original magnification $\times 1000$; immersion objective $\times 100 / \times 100$ with immersion oil type). Images are representative of three independent experiments.

wogonin for $5 \mathrm{~d}$ in Hut-102 and Jurkat cells (Figures 7(a) and 7(b)). We chose Jurkat cells to assess cell viability affected by Navitoclax. The CCK8 assay indicated that wogonin-induced senescent cells exhibited more significant sensitivity to Navi- toclax than control cells (Figure 7(c)). Meanwhile, cell apoptosis analyses showed that Navitoclax could induce more apoptosis in wogonin-treated cells than in untreated cells (Figure $7(d)$ ). Moreover, western blot results showed that 


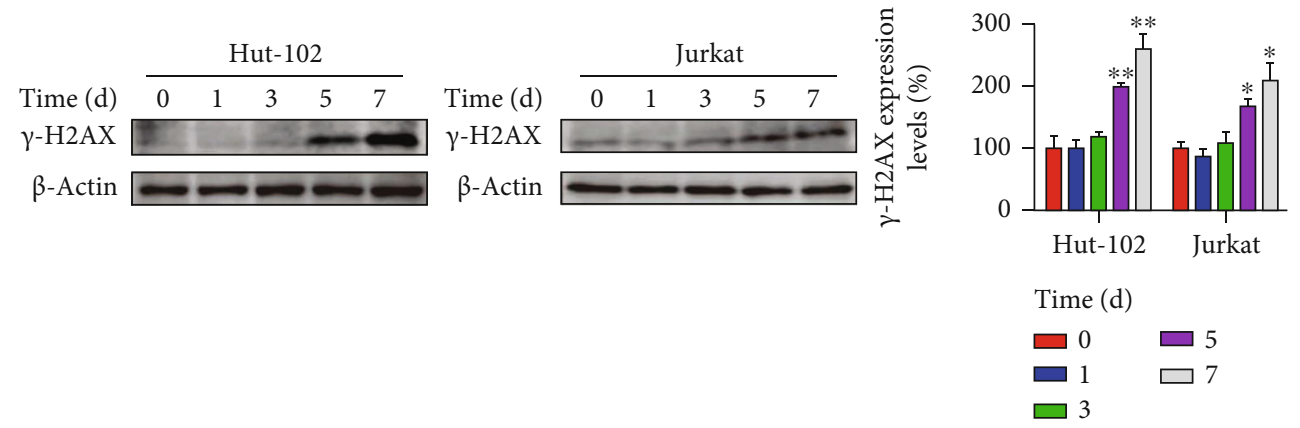

(a)
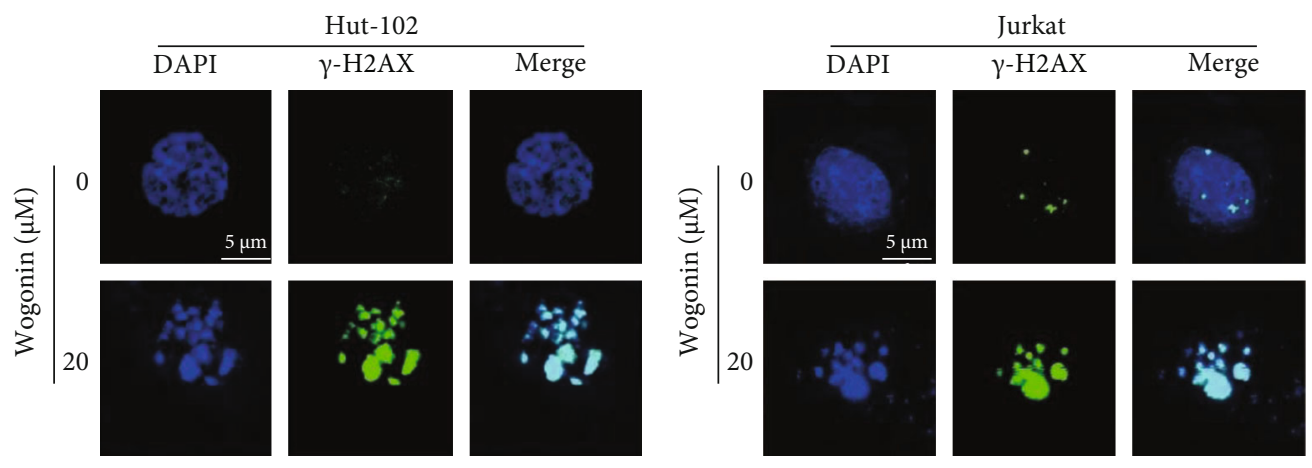

(b)
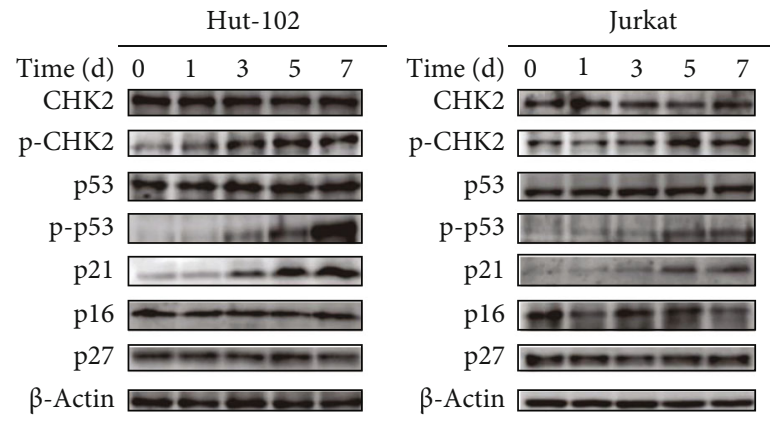

(c)

Figure 6: Continued. 

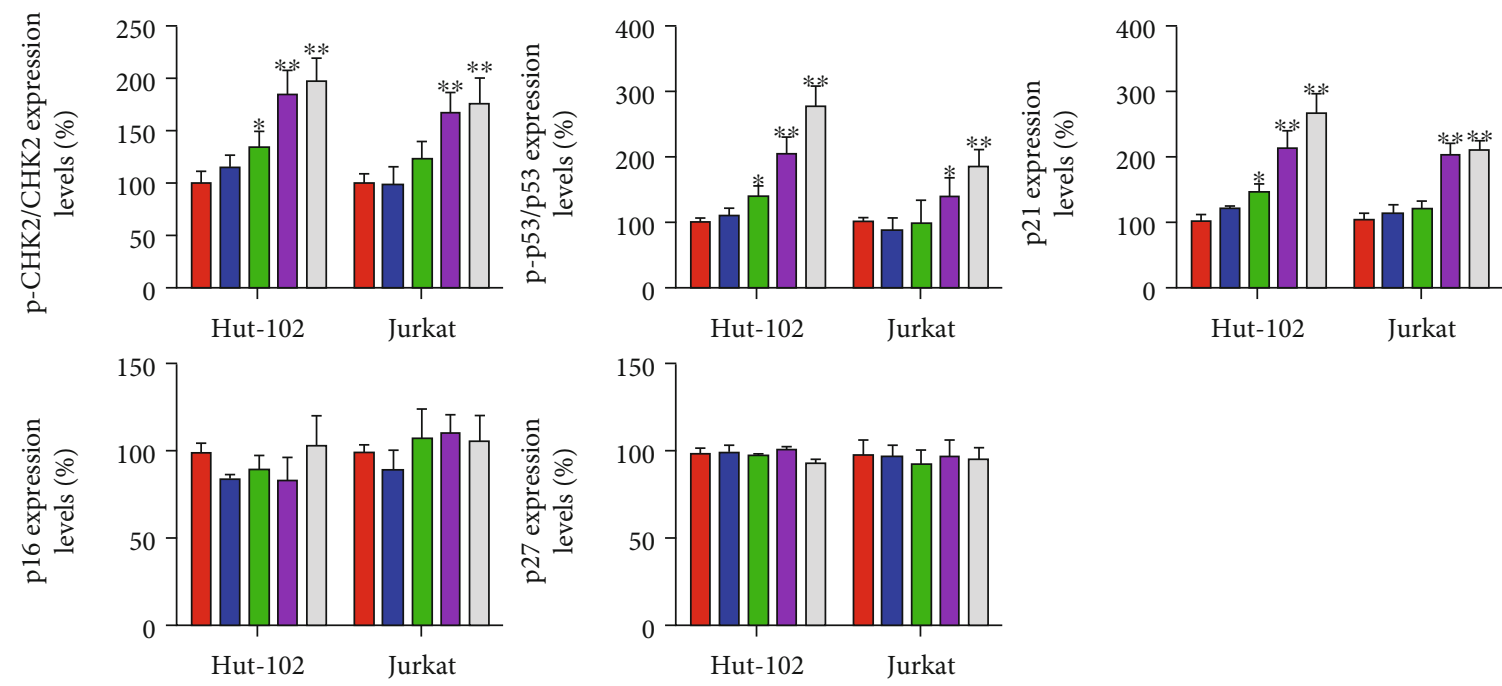

$$
\begin{array}{ll}
\text { Time (d) } & \\
\square 0 & \square 5 \\
{ }_{1} & \square 7 \\
\square 3 &
\end{array}
$$

(d)

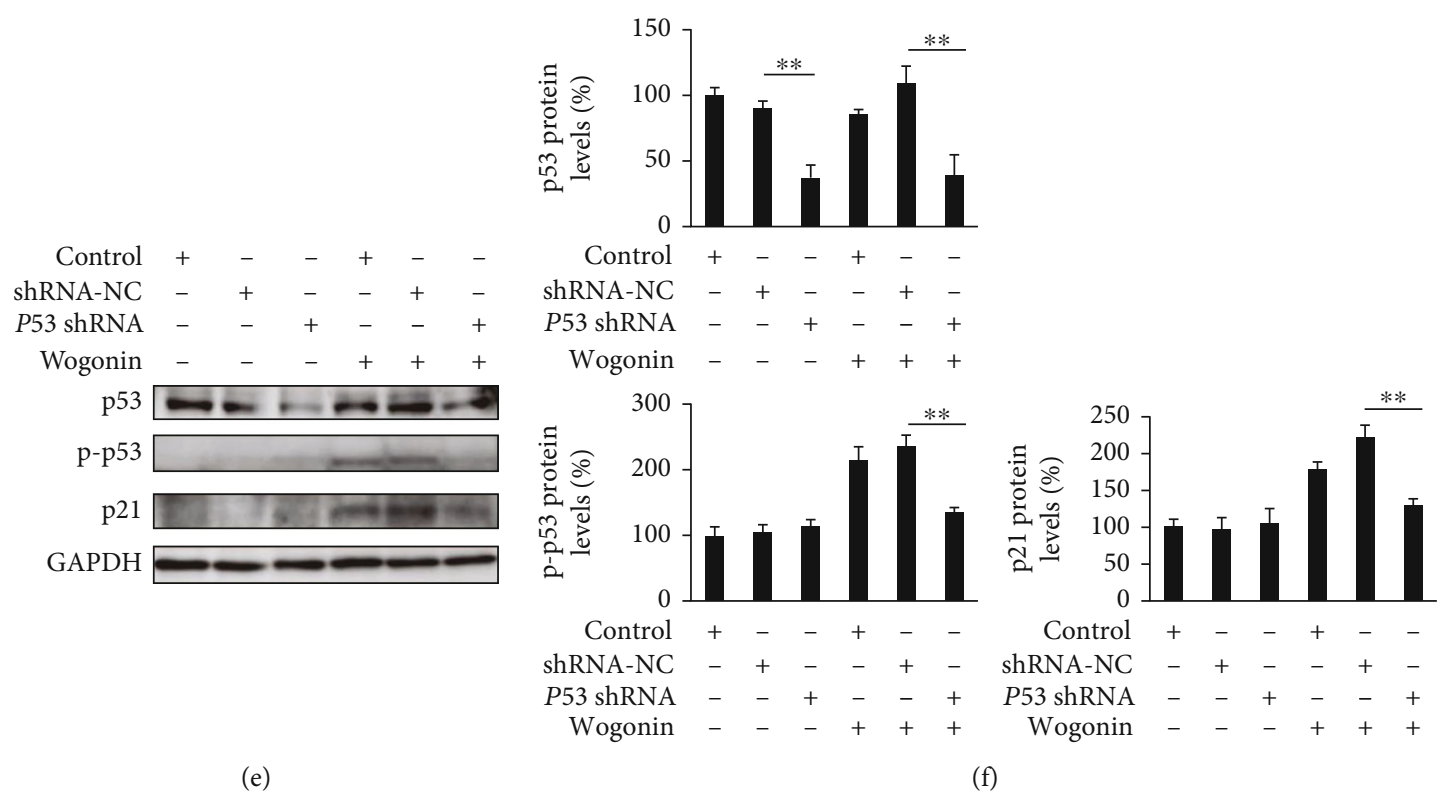

Figure 6: Continued. 


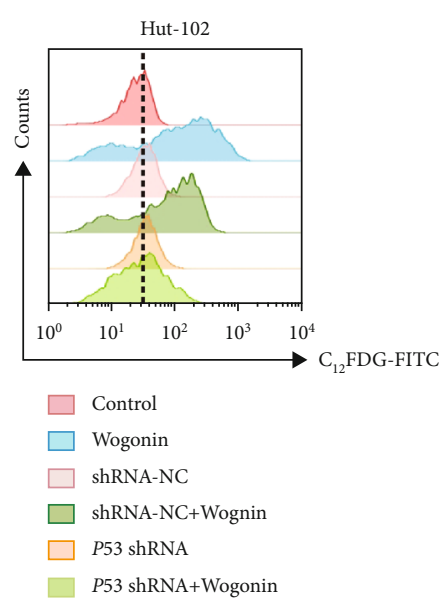

(g)

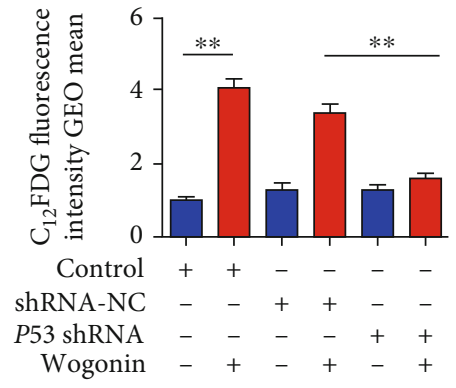

(h)

FIGURE 6: Wogonin induced cellular senescence through p53-mediated DNA damage response. (a) Hut-102 and Jurkat cells were treated with or without $20 \mu \mathrm{M}$ wogonin for the indicated time periods, and western blot was used to detect the expression change of the DNA damage marker protein $\gamma$-H2AX. $\beta$-Actin was used as loading controls. Relative protein expression level of $\gamma$-H2AX was determined. Columns represent the mean from three parallel experiments (mean \pm SEM). ${ }^{*} p<0.05,{ }^{* *} p<0.01$, compared with the control group. (b) Hut-102 and Jurkat cells were treated with or without $20 \mu \mathrm{M}$ wogonin for $5 \mathrm{~d}$; then, the cells were fixed, permeabilized, and stained with an antibody against $\gamma$-H2AX (green), while nuclei were stained with DAPI (blue). Immunofluorescent images showed the distribution of $\gamma$ H2AX in Hut-102 and Jurkat cells (original magnification $\times 1000$; immersion objective $\times 100 / \times 100$ with immersion oil type). Images are representative of three independent experiments. (c) Hut-102 and Jurkat cells were treated with or without $20 \mu \mathrm{M}$ wogonin for the indicated time periods $(0,1,3,5$, and $7 \mathrm{~d}$ ), and western blot was performed to detect the expression changes of CHK2, p-CHK2 (T68), p53, p-p53 (Ser15), p21, p16, and p27. $\beta$-Actin was used as loading controls. (d) Relative protein expression levels of p-CHK2/CHK2, pp53/p53, p21, p16, and p27 were determined. Columns represent the mean from three parallel experiments $\left(\right.$ mean \pm SEM). ${ }^{*} p<0.05,{ }^{* *} p$ $<0.01$, compared with the control group. (e) The protein expression levels of p53, p-p53 (Ser15), and p21 were detected by western blot. P53 shRNA-transfected Hut-102, shRNA-NC, and control Hut-102 cells were treated with or without $20 \mu \mathrm{M}$ wogonin for 5 d. GAPDH was used as loading controls. (f) Relative protein expression levels of p53, p-p53 (Ser15), and p21. Columns represent the mean from three parallel experiments (mean $\pm \mathrm{SEM}$ ). ${ }^{*} p<0.05,{ }^{* *} p<0.01$, compared with the control group. (g) Hut-102 cells were transfected with P53 shRNA and shRNA-NC by a lentiviral vector. P53 shRNA-transfected Hut-102, shRNA-NC, and control Hut-102 cells were treated with or without $20 \mu \mathrm{M}$ wogonin for $5 \mathrm{~d}$. The activity of SA- $\beta$-Gal was measured by flow cytometry (cells were stained with $\mathrm{C}_{12} \mathrm{FDG}$ ). (h) Relative SA- $\beta$-Gal activity was assessed by using the GEOmean of groups, which represented $\mathrm{C}_{12}$ FDG fluorescence intensity. Columns represent the mean from three parallel experiments (mean $\pm \mathrm{SEM}$ ). ${ }^{*} p<0.05,{ }^{* *} p<0.01$, compared with the NC group.

the expression of apoptosis protein levels like cleaved caspase-3/caspase-3, cleaved PARP-1/PARP-1, Bax, and Bim $_{\mathrm{L}}$ was increased in Navitoclax-treated senescent cells (Figures 7(e) and 7(f)). In conclusion, wogonin-induced senescent cells were more sensitive to Navitoclax cytotoxicity and were effectively killed.

\section{Discussion}

T-cell malignancies remain to have poor prognosis and are difficult to have specific treatments $[3,52]$. Clinically, chemotherapy based on the ABVD and CHOP regimen is still the preferred treatment for $\mathrm{T}$-cell malignancies, and toxic effects and drug resistance brought by traditional chemotherapy regimens are unavoidable [53-55]. Cellular senescence makes senescent cells as a druggable target to repress tumor progression [22, 56]. Thus, inducing cellular senescence makes senescent cells display an induced vulnerability to senolytics drugs, which could be a novel one-two punch therapy for the treatment of T-cell malignancies [19, 50, 57].

TP53 mutations in hematologic malignancies are less frequent (5\%-15\%) but closely associated with poor prognosis [58]. Different sites, forms, and frequencies of TP53 mutation lead to different disease states [58-60]. Because of the central role of p53 in regulating cells' normal life activity, drug discovery concentrates on manipulating p53 and eradicating cancer cells. Wogonin is a well-known flavonoid and has a wide range of anticancer effects especially producing a better effect in hematologic malignancies [61-64]. However, although moderate and high concentrations of wogonin (over $40 \mu \mathrm{M}$ ) had a better therapeutic effect, its insoluble characteristic and cytotoxicity in high doses were inescapable and limited its use in clinic. We found that low concentration of wogonin $(20 \mu \mathrm{M})$ could induce cellular senescence via DNA damage response in T-cell malignancies and verified the application of one-two punch therapy involving cellular senescence. Targeting senescent cells may benefit to increase therapeutic efficacy and reduce risk of recurrence [19]. Moreover, a previous study also reported that wogonin could target the Warburg Effect which depends on wild-type p53 to produce anticancer effects [65]. Except in the field of metabolism, p53 also plays an important role in regulating cellular senescence [66]. Our results showed that wogonin induced cellular senescence in p53 functional cell lines (Hut-102, Jurkat) but not the p53-deficient Hut-78 cell line. And we also found senescence-related SAHF phenomenon in Hut-102 

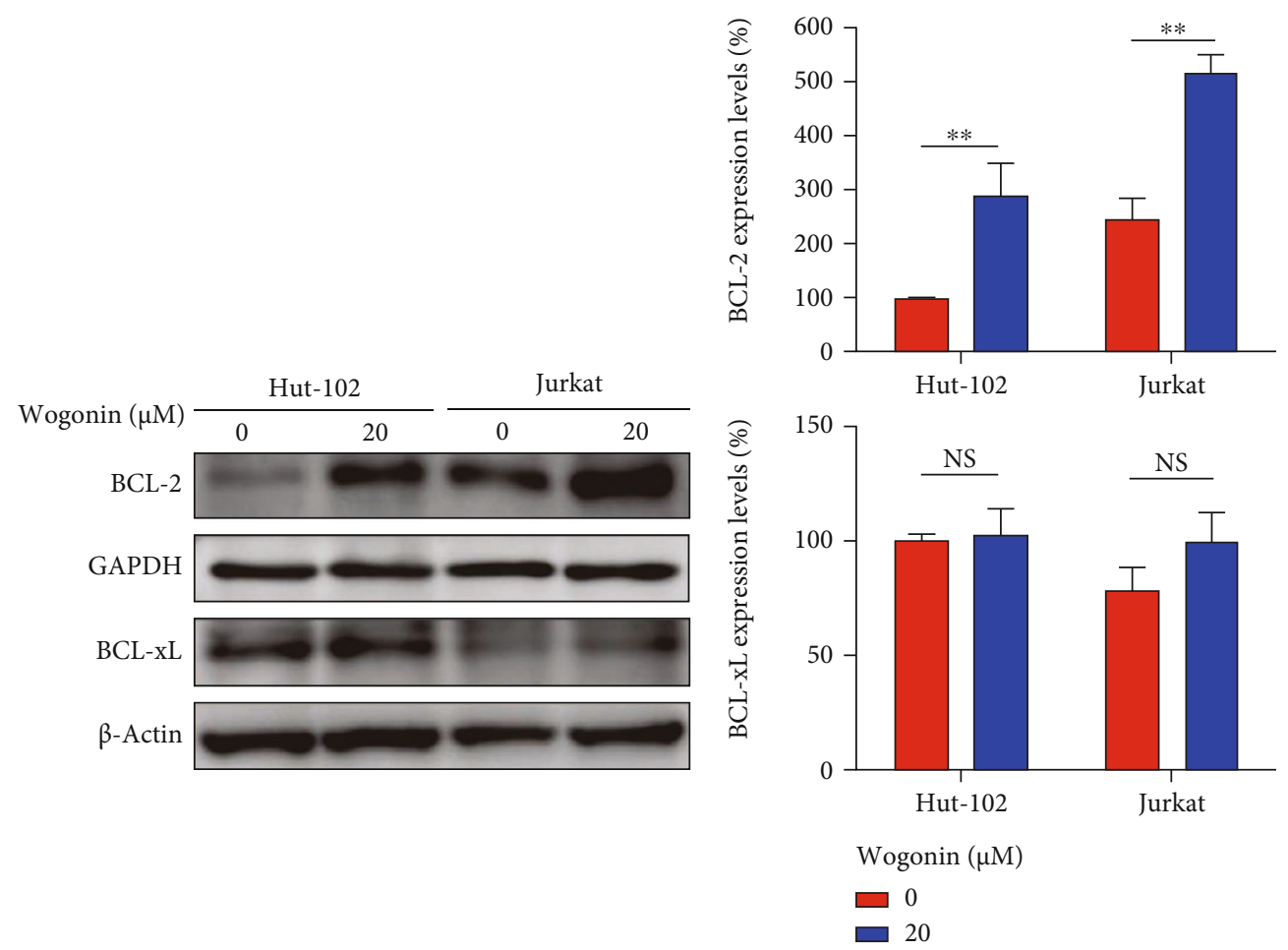

(a)

(b)
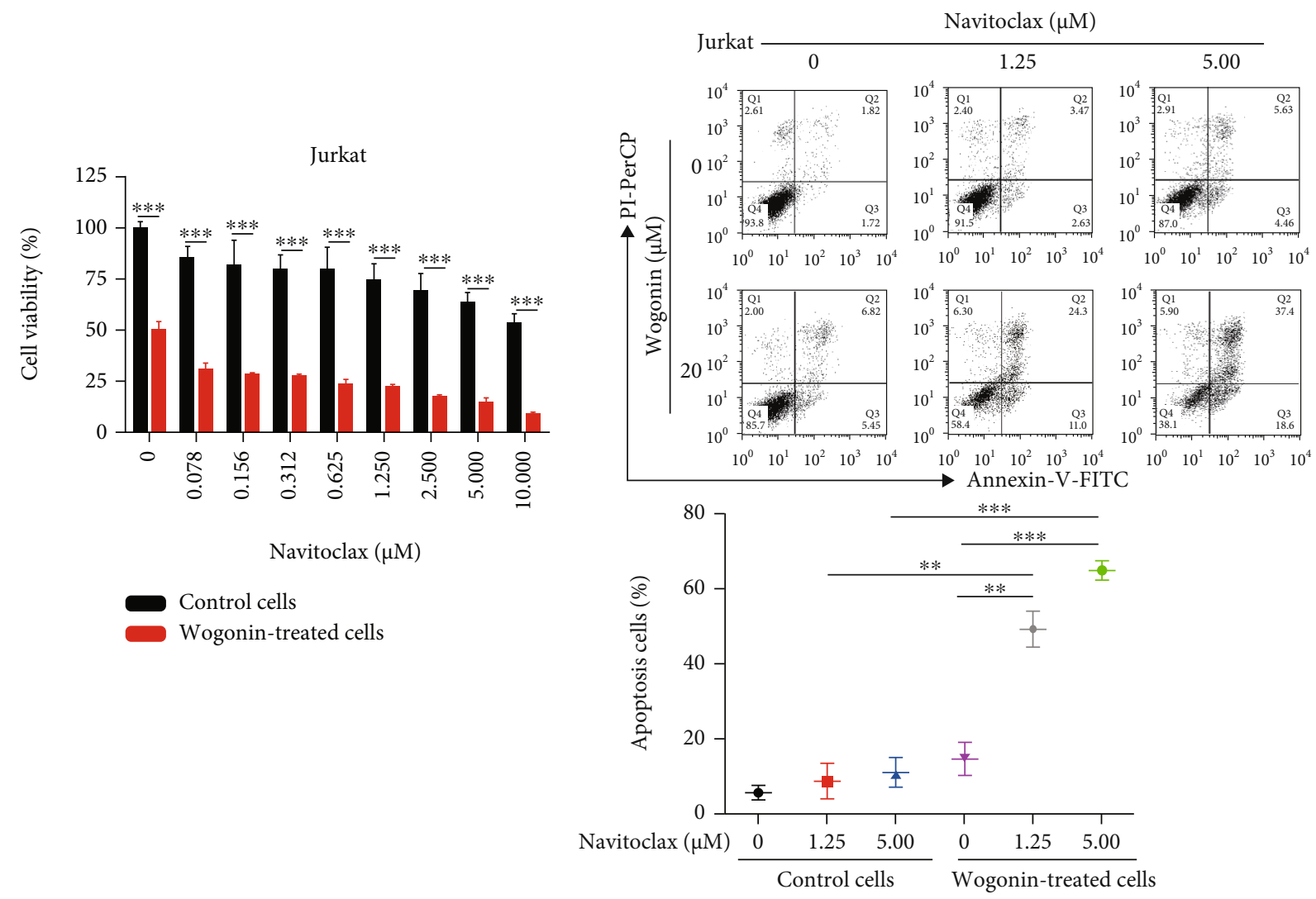

(c)

(d)

Figure 7: Continued. 


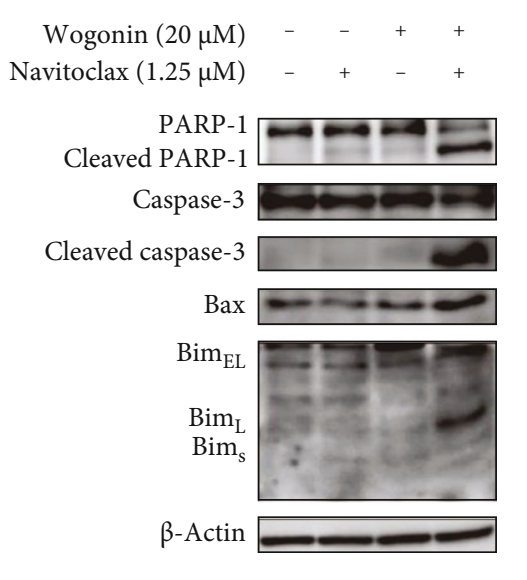

(e)

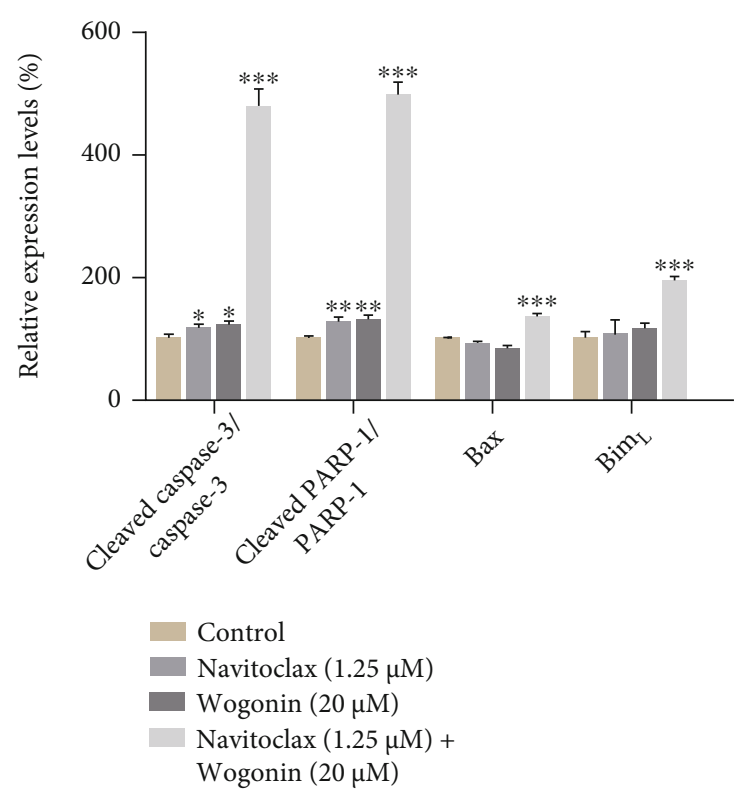

$(\mathrm{f})$

FIgURe 7: Wogonin-induced cellular senescence sensitized Navitoclax to kill T-cell malignancy cells. (a) Hut-102 and Jurkat cells were treated with or without $20 \mu \mathrm{M}$ wogonin for $5 \mathrm{~d}$, and western blot was used to detect the expression change of BCL- 2 and BCL-xL. GAPDH and $\beta$ actin were used as loading controls. (b) Relative protein expression levels of BCL-2 and BCL-xL. Columns represent the mean from three parallel experiments (mean $\pm \mathrm{SEM}$ ). ${ }^{*} p<0.05,{ }^{* *} p<0.01$, compared with the control group. (c) Jurkat cells were exposed to $20 \mu \mathrm{M}$ wogonin for $5 \mathrm{~d}$, and then, control or wogonin-treated Jurkat cells were treated with the indicated concentrations of Navitoclax $(0-10 \mu \mathrm{M})$ for $48 \mathrm{~h}$. Cell viability was assayed by CCK8. The data represent the mean \pm SEM of three different experiments. ${ }^{* * *} p<0.001$, compared with the control group. (d) Jurkat cells were exposed to $20 \mu \mathrm{M}$ wogonin for $5 \mathrm{~d}$, and then, control or wogonin-treated Jurkat cells were then treated with the indicated concentrations of Navitoclax $(1.25,5 \mu \mathrm{M})$ for $48 \mathrm{~h}$. Cell apoptosis was assayed by flow cytometry after Annexin-V/PI staining. Data represent the mean from three parallel experiments (mean \pm SEM). ${ }^{*} p<0.05,{ }^{* *} p<0.01$, and ${ }^{* * *} p<0.001$, compared with the control group. (e) Jurkat cells were exposed to $20 \mu \mathrm{M}$ wogonin for $5 \mathrm{~d}$, and then, control or wogonin-treated Jurkat cells were then treated with the indicated concentrations of Navitoclax $(1.25 \mu \mathrm{M})$ for $48 \mathrm{~h}$. The protein expression levels of PARP-1, cleaved PARP-1, caspase-3, cleaved caspase-3, Bax, and Bim were analyzed by western blot. $\beta$-Actin were used as loading controls. (f)

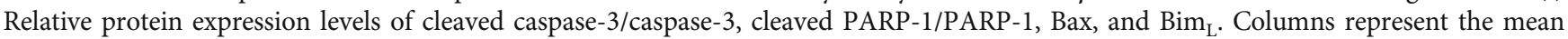
from three parallel experiments (mean $\pm \mathrm{SEM}$ ). ${ }^{*} p<0.05,{ }^{* *} p<0.01$, and ${ }^{* * *} p<0.001$, compared with the control group.

and Jurkat cells but not in Hut-78 cells, suggesting that p53 may be involved in wogonin-induced senescence. Meanwhile, the phosphorylation of p53 (Ser15) was increased in senescent cells after the treatment with wogonin for $5 \mathrm{~d}$. The knocking down assay confirmed that wogonin-induced senescence depended on the expression of p53. It is indicated that p53 may be a core factor in mechanisms of wogonintreated T-cell malignancies.

According to our study, wogonin induced DNA damage response and increased the expression of $\gamma$-H2AX in Hut102 and Jurkat cells. As the consequences of DNA damage response, phosphorylation of CHK2 (Thr68) was activated after the treatment with wogonin in senescent cells. The mRNA and protein expression of p21 playing a crucial role was obviously increased by wogonin treatment to render the cell cycle arrest irreversible and induce the senescence phenotype. The mRNA level of p27 did not change in Hut-102 and Jurkat cells, and the mRNA level of p16 had slightly changed in Jurkat cells but not in Hut-102 cells. It is also worth noting that the protein expression of p16 did not change in Hut-102 and Jurkat cells, and the results prompted us that the $\mathrm{p} 16 / \mathrm{Rb}$ pathway may not mainly participate in wogonin-induced cellular senescence in Tcell malignancies. In a word, the results suggested that the CHK2/p53/p21 pathway may be involved in wogonininduced cellular senescence.

DNA damage is only the mediator of cellular senescence, but the change of telomere function is one of the real causes to trigger DNA damage response resulting in cellular senescence [67]. Telomere dysfunction leads to aging, which is a proliferation disorder dependent on p53 and pRb, and hinders the continuous accumulation of mutations, thus resulting in blocking the development of cancer. In contrast, experimental inactivation of $\mathrm{p} 53$ and pRb enables fibroblasts to bypass aging and continue to divide $[68,69]$. As a mild senescence inducer, nonlethal concentration of wogonin showed a unique function to T-cell malignancies. $20 \mu \mathrm{M}$ wogonin obviously suppressed the transcription of $C-M Y C$ and its downstream gene $h T E R T$. Furthermore, wogonin decreased the protein expression of c-Myc and hTERT. $C$ $M Y C$ is a well-known oncogene and regulates the occurrence and progression of cancer. The promoter region of hTERT has oncoprotein c-Myc binding site-E-boxes, which induce the transcriptional activity of $h T E R T$ and activate telomerase 


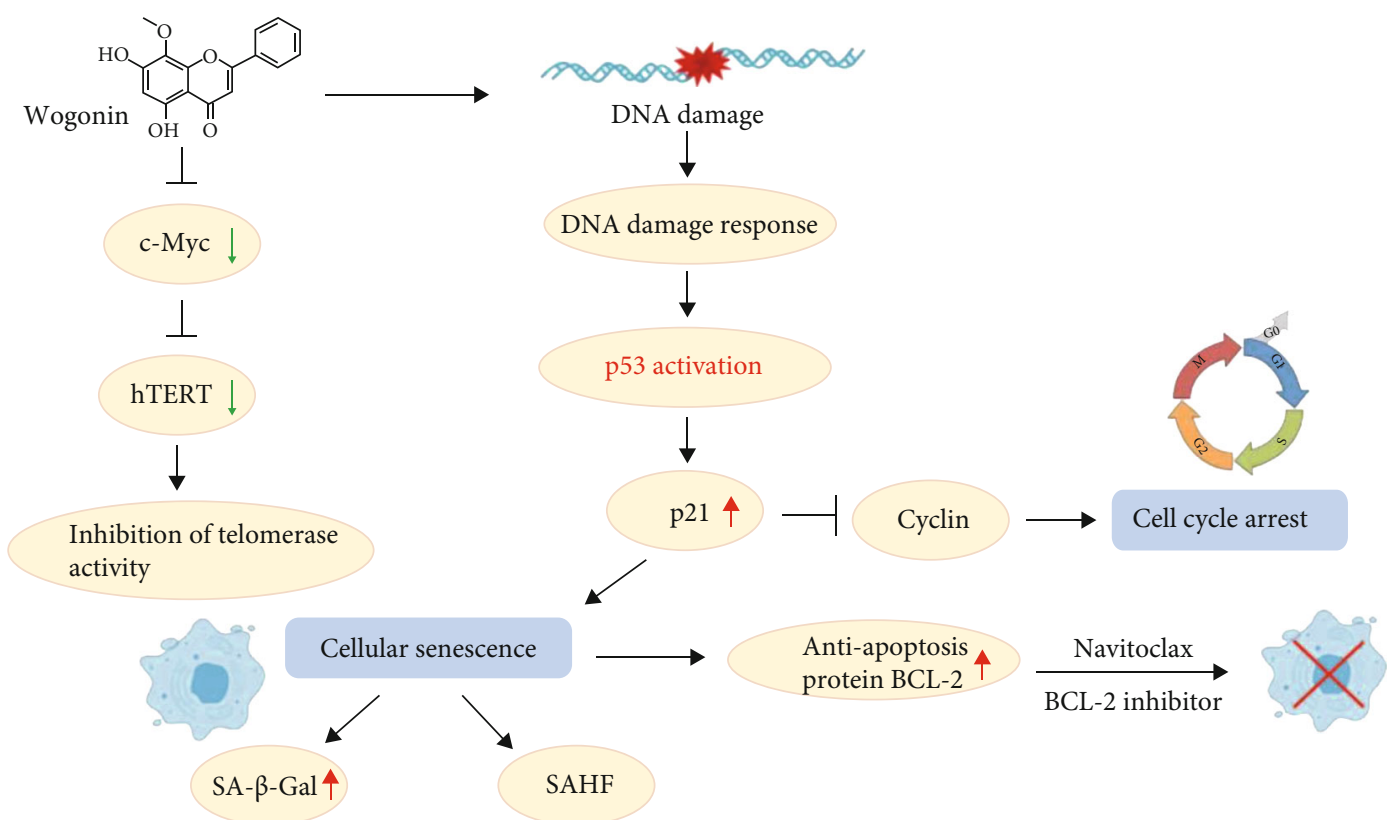

Figure 8: Graphical abstract on the mechanisms of one-two punch therapy involved in wogonin-induced cellular senescence in T-cell malignancies.

[70]. We can infer that wogonin induced cellular senescence partly through the inhibition of telomere function. The underlying mechanisms are still being investigated.

In this paper, we used Hut-78 cells as the p53 deficiency model to verify the mechanism of wogonin. We did not observe obvious cellular senescence in Hut-78 cells after wogonin treatment. It is worth mentioning that we found that wogonin increased the level of $\gamma$-H2AX in Hut-78 cells (Figure S1a, b). Following the results, we detected the expression of CHK2, p-CHK2 (T68), p21, p16, and p27. The results showed that the expression of $\mathrm{CHK} 2$ and $\mathrm{p}$ CHK2 (T68) were all increased after wogonin treatment for $7 \mathrm{~d}$. The expression of p21 had slightly increased on day 7 , but p16 and p27 had no change (Figure S1c, d). The upregulated folds of p21 in Hut-102 and Jurkat cells were more than those in Hut-78 cells. The expression of p21 was slightly increased in Hut-78 cells after treatment with wogonin slighter (Figure 6(d) and Figure S1d). However, the lack of p53 and slightly increased level of p21 may not be enough give rise to senescence in Hut-78 cells. CFSE experiments suggested that the decreased fluorescence intensity in CFSE-labeled cells was prevented by wogonin in Hut-78 cells. Thus, we supposed that there could be other signaling pathways to account for the mechanism which regulates cell proliferation induced by wogonin in Hut-78 cells. More follow-up studies are warranted to unveil the true nature of wogonin in Hut-78 cells.

Some studies have shown that overexpression of BCL-2 or BCL-xL is commonly observed in acute lymphoblastic leukemia (ALL) and chronic lymphocytic leukemia (CLL) [71, 72]. Navitoclax is a specific $\mathrm{BH} 3$-mimetic drug especially used in hematologic malignancies [73]. However, BCL-2 was verified as playing a key role in lymphoid cells, and overexpression of BCL-2 could increase the sensibility to Navito- clax [51]. But lymphoid cells with low expression of BCL-2 lack sensibility to Navitoclax and lose opportunity for optimal cell killing. The expression of BCL-2 was upregulated, contributing to the resistance of senescent cells to death. In our study, the increased expression of BCL-2 in senescent cells induced by wogonin could be detected by western blot. Then, the CCK8 assay showed that senescent cells were more sensitive to Navitoclax compared to untreated cells. Thus, the sequential combination of the BCL-2 inhibitor Navitoclax could promote the death of tumor cells, so there was no doubt that adopting this treatment method was of positive significance.

In summary, our findings suggest that wogonin not only inhibited telomere function but also induced p53-dependent cellular senescence, which was correlated with DDR. Cellular senescence promoted the expression of BCL-2 and consequently increased the sensibility to Navitoclax and removed senescent cells. Our work indicates prosenescence therapy induced by wogonin, sequentially combined with Navitoclax, providing a novel clinical approach for T-cell malignancy treatment (Figure 8).

\section{Conclusion}

In conclusion, our study revealed that wogonin inhibited telomerase activity via inhibition of $\mathrm{c}-\mathrm{Myc}$ regulating the expression of hTERT. On this basis, we also found that wogonin induced p53-dependent cellular senescence mediated by DDR in T-cell malignancies. Moreover, wogonin-induced senescent cells increased the sensibility of Navitoclax resulting in killing T-cell malignancy cells effectively. cells. This may be considered a promising aspect of clinical cancer treatment. 


\section{Data Availability}

The authors declare that the data supporting the findings of this study are available from the corresponding author upon reasonable request.

\section{Additional Points}

Additional Information. The online version of this article contains supplementary material, which is available to authorized users.

\section{Conflicts of Interest}

The authors declare that they have no conflicts of interest.

\section{Authors' Contributions}

YJQ and HL designed and performed the research, analyzed the data, and wrote the paper. YZZ and $\mathrm{PH}$ performed the research and analyzed the data. XYW and XXY performed the research. MYZ and HZW collected the data and performed statistical analysis. ZYW collected and analyzed the data, and QLG and $\mathrm{HH}$ conceptualized the project and directed the experimental design data analysis. Yingjie Qing and Hui Li contributed equally to this work.

\section{Acknowledgments}

The work was supported by the National Natural Science Foundation of China (81873046, 81830105, 81903647, 81503096 , and 81673461), the Drug Innovation Major Project (2017ZX09301014, 2018ZX09711001-003-007, and 2017ZX09101003-005-023), the Natural Science Foundation of Jiangsu Province (BK20190560, BE2018711), the Nanjing Medical Science and Technology Development Project (YKK17074, YKK19064), the Research and Innovation Project for College Graduates of Jiangsu Province (KYCX18_0803), the China Postdoctoral Science Foundation (No. 2018M642373), and the "Double First-Class" University Project (CPU2018GF11, CPU2018GF05).

\section{Supplementary Materials}

Supplemental Figure. Effect of $20 \mu \mathrm{M}$ wogonin on Hut-78 cell line. (a) Hut-78 cells were treated with or without $20 \mu \mathrm{M}$ wogonin for the indicated time periods $(0,1,3,5,7$ $\mathrm{d})$, and western blot was used to detect the changes in the expression of DNA damage marker protein $\gamma$-H2AX. $\beta$-actin was used as loading controls. The right panel: relative protein expression level of $\gamma-\mathrm{H} 2 \mathrm{AX}$. Columns represent the mean from three parallel experiments (mean \pm SEM). ${ }^{*} p<0.05,{ }^{* *}$ $p<0.01$, compared with control group. (b) Hut-78 cells were treated with or without $20 \mu \mathrm{M}$ wogonin for $7 \mathrm{~d}$, then the cells were fixed, permeabilized and stained with antibody against $\gamma$-H2AX (Green), while nuclei were stained with DAPI (Blue). Immunofluorescent images showed the distribution of $\gamma$-H2AX in Hut-78 cells (original magnification $\times 1000$; immersion objective $\times 100 / \times 100$ with immersion oil type). Images are representative of three independent experiments. (c) Hut-78 cells were treated with or without $20 \mu \mathrm{M}$ wogonin for the indicated time periods $(0,1,3,5,7 \mathrm{~d})$, and western blot was performed to detect the changes in the expression of CHK2, p-CHK2 (T68), p21, p16 and p27. $\beta$-actin was used as loading controls. (d) Relative protein levels of $\mathrm{p}$ CHK2/CHK2, p16, p21 and p27 were determined. Columns represent the mean from three parallel experiments (mean \pm SEM). ${ }^{*} p<0.05,{ }^{* *} p<0.01$, compared with control group. (Supplementary Materials)

\section{References}

[1] The Non-Hodgkin's Lymphoma Classification Project, "A clinical evaluation of the International Lymphoma Study Group classification of non-Hodgkin's lymphoma," Blood, vol. 89, no. 11, pp. 3909-3918, 1997.

[2] I. Aifantis, E. Raetz, and S. Buonamici, "Molecular pathogenesis of T-cell leukaemia and lymphoma," Nature reviews Immunology, vol. 8, no. 5, pp. 380-390, 2008.

[3] P. M. Maciocia, P. A. Wawrzyniecka, B. Philip et al., "Targeting the $\mathrm{T}$ cell receptor $\beta$-chain constant region for immunotherapy of T cell malignancies," Nature Medicine, vol. 23, no. 12, pp. 1416-1423, 2017.

[4] M. Mamonkin, R. H. Rouce, H. Tashiro, and M. K. Brenner, "A T-cell-directed chimeric antigen receptor for the selective treatment of T-cell malignancies," Blood, vol. 126, no. 8, pp. 983-992, 2015.

[5] T. Palomero and A. Ferrando, "Targeted cellular immunotherapy for T cell malignancies," Nature Medicine, vol. 23, no. 12, pp. 1402-1403, 2017.

[6] J. A. Ross, M. Spadaro, D. C. Rosado, F. Cavallo, R. A. Kirken, and F. Pericle, "Inhibition of JAK3 with a novel, selective and orally active small molecule induces therapeutic response in T-cell malignancies," Leukemia, vol. 28, no. 4, pp. 941-944, 2014.

[7] S. Deng, S. Lin, J. Shen, and Y. Zeng, "Comparison of CHOP vs CHOPE for treatment of peripheral T-cell lymphoma: a metaanalysis," Oncotargets and Therapy, vol. 12, pp. 2335-2342, 2019.

[8] B. Jia, S. Hu, J. Yang et al., "Comparison of gemcitabin, cisplatin, and dexamethasone (GDP), CHOP, and CHOPE in the first-line treatment of peripheral T-cell lymphomas," Hematology, vol. 21, no. 9, pp. 536-541, 2016.

[9] K. Laribi, M. Alani, C. Truong, and A. Baugier de Materre, "Recent advances in the treatment of peripheral T-cell lymphoma," The Oncologist, vol. 23, no. 9, pp. 1039-1053, 2018.

[10] A. Broccoli and P. L. Zinzani, "Peripheral T-cell lymphoma, not otherwise specified," Blood, vol. 129, no. 9, pp. 11031112, 2017.

[11] M. Demaria, M. N. O'Leary, J. Chang et al., "Cellular senescence promotes adverse effects of chemotherapy and cancer relapse," Cancer Discovery, vol. 7, no. 2, pp. 165-176, 2017.

[12] I. Ben-Porath and R. A. Weinberg, "The signals and pathways activating cellular senescence," The International Journal of Biochemistry \& Cell Biology, vol. 37, no. 5, pp. 961-976, 2005.

[13] L. Zhao, Y. Zhang, Y. Gao et al., "JMJD3 promotes SAHF formation in senescent WI38 cells by triggering an interplay between demethylation and phosphorylation of RB protein," Cell Death and Differentiation, vol. 22, no. 10, pp. 16301640, 2015. 
[14] Y. Pan, M. Meng, N. Zheng et al., "Targeting of multiple senescence-promoting genes and signaling pathways by triptonide induces complete senescence of acute myeloid leukemia cells," Biochemical Pharmacology, vol. 126, pp. 34-50, 2017.

[15] Y. Qin, H. Guo, B. Tang, and S. M. Yang, "The non-reverse transcriptase activity of the human telomerase reverse transcriptase promotes tumor progression (review)," International Journal of Oncology, vol. 45, no. 2, pp. 525-531, 2014.

[16] Y. Sun, J. Campisi, C. Higano et al., "Treatment-induced damage to the tumor microenvironment promotes prostate cancer therapy resistance through WNT16B," Nature Medicine, vol. 18, no. 9, pp. 1359-1368, 2012.

[17] M. Milanovic, D. N. Y. Fan, D. Belenki et al., "Senescence-associated reprogramming promotes cancer stemness," Nature, vol. 553, no. 7686, pp. 96-100, 2018.

[18] L. Wang, R. Leite de Oliveira, C. Wang et al., "High-throughput functional genetic and compound screens identify targets for senescence induction in cancer," Cell Reports, vol. 21, no. 3, pp. 773-783, 2017.

[19] C. J. Sieben, I. Sturmlechner, B. van de Sluis, and J. M. van Deursen, "Two-step senescence-focused cancer therapies," Trends in Cell Biology, vol. 28, no. 9, pp. 723-737, 2018.

[20] C. Wang, S. Vegna, H. Jin et al., "Inducing and exploiting vulnerabilities for the treatment of liver cancer," Nature, vol. 574, no. 7777, pp. 268-272, 2019.

[21] Y. Zhu, T. Tchkonia, H. Fuhrmann-Stroissnigg et al., "Identification of a novel senolytic agent, navitoclax, targeting the Bcl-2 family of anti-apoptotic factors," Aging Cell, vol. 15, no. 3, pp. 428-435, 2016.

[22] B. G. Childs, M. Gluscevic, D. J. Baker et al., "Senescent cells: an emerging target for diseases of ageing," Nature Reviews Drug Discovery, vol. 16, no. 10, pp. 718-735, 2017.

[23] T. R. Kasparek and T. C. Humphrey, "DNA double-strand break repair pathways, chromosomal rearrangements and cancer," Seminars in Cell \& Developmental Biology, vol. 22, no. 8, pp. 886-897, 2011.

[24] H. L. Ou and B. Schumacher, "DNA damage responses and p53 in the aging process," Blood, vol. 131, no. 5, pp. 488-495, 2018.

[25] S. Salmoiraghi, A. Rambaldi, and O. Spinelli, "TP53 in adult acute lymphoblastic leukemia," Leukemia \& Lymphoma, vol. 59, no. 4, pp. 778-789, 2018.

[26] Y. Shiloh, "ATM and related protein kinases: safeguarding genome integrity," Nature Reviews. Cancer, vol. 3, no. 3, pp. 155-168, 2003.

[27] Y. H. Ou, P. H. Chung, T. P. Sun, and S. Y. Shieh, "p53 Cterminal phosphorylation by $\mathrm{CHK} 1$ and $\mathrm{CHK} 2$ participates in the regulation of DNA-damage-induced C-terminal acetylation," Molecular Biology of the Cell, vol. 16, no. 4, pp. 16841695, 2005.

[28] R. Mirzayans, B. Andrais, A. Scott, and D. Murray, "New insights into p53 signaling and cancer cell response to DNA damage: implications for cancer therapy," Journal of Biomedicine \& Biotechnology, vol. 2012, Article ID 170325, 16 pages, 2012.

[29] M. C. Tai, S. Y. Tsang, L. Y. Chang, and H. Xue, "Therapeutic potential of wogonin: a naturally occurring flavonoid," CNS Drug Reviews, vol. 11, no. 2, pp. 141-150, 2005.

[30] Y. Chen, H. Hui, H. Yang et al., "Wogonoside induces cell cycle arrest and differentiation by affecting expression and subcellular localization of PLSCR1 in AML cells," Blood, vol. 121, no. 18, pp. 3682-3691, 2013.
[31] K. M. Hui, M. S. Huen, H. Y. Wang et al., "Anxiolytic effect of wogonin, a benzodiazepine receptor ligand isolated from _Scutellaria baicalensis_Georgi," Biochemical Pharmacology, vol. 64, no. 9, pp. 1415-1424, 2002.

[32] X. Yu, H. Li, M. Zhu et al., "Involvement of p53 acetylation in growth suppression of cutaneous T-cell lymphomas induced by HDAC inhibition," The Journal of Investigative Dermatology, vol. 140, no. 10, pp. 2009-2022.e4, 2020.

[33] M. A. Sliwinska, G. Mosieniak, K. Wolanin et al., "Induction of senescence with doxorubicin leads to increased genomic instability of HCT116 cells," Mechanisms of Ageing and Development, vol. 130, no. 1-2, pp. 24-32, 2009.

[34] N. V. Petrova, A. K. Velichko, S. V. Razin, and O. L. Kantidze, "Small molecule compounds that induce cellular senescence," Aging Cell, vol. 15, no. 6, pp. 999-1017, 2016.

[35] H. Jin, N. Lian, F. Zhang et al., "Activation of PPAR_ $\gamma_{-} /$P53 signaling is required for curcumin to induce hepatic stellate cell senescence," Cell Death \& Disease, vol. 7, no. 4, article e2189, 2016.

[36] F. d'Adda di Fagagna, "Living on a break: cellular senescence as a DNA-damage response," Nature Reviews Cancer, vol. 8, no. 7, pp. 512-522, 2008.

[37] M. B. Kastan and J. Bartek, "Cell-cycle checkpoints and cancer," Nature, vol. 432, no. 7015, pp. 316-323, 2004.

[38] S. H. Park, J. S. Lim, and K. L. Jang, "All-trans retinoic acid induces cellular senescence via upregulation of p16, p21, and p27," Cancer Letters, vol. 310, no. 2, pp. 232-239, 2011.

[39] M. Y. Terzi, M. Izmirli, and B. Gogebakan, "The cell fate: senescence or quiescence," Molecular Biology Reports, vol. 43, no. 11, pp. 1213-1220, 2016.

[40] J. Liu, L. Wang, Z. Wang, and J. P. Liu, "Roles of telomere biology in cell senescence, replicative and chronological ageing," Cells, vol. 8, no. 1, p. 54, 2019.

[41] M. A. Blasco, "Telomeres and human disease: ageing, cancer and beyond," Nature Reviews. Genetics, vol. 6, no. 8, pp. 611622, 2005.

[42] M. Bellon and C. Nicot, "Multiple pathways control the reactivation of telomerase in HTLV-I-associated leukemia," International journal of cancer and oncology, vol. 2, no. 2, pp. 1-8, 2015.

[43] K. C. Low and V. Tergaonkar, "Telomerase: central regulator of all of the hallmarks of cancer," Trends in Biochemical Sciences, vol. 38, no. 9, pp. 426-434, 2013.

[44] Y. Zhang, M. Sun, W. Shi et al., "Arsenic trioxide suppresses transcription of hTERT through down-regulation of multiple transcription factors in HL-60 leukemia cells," Toxicology Letters, vol. 232, no. 2, pp. 481-489, 2015.

[45] J. S. Lee, Y. Mo, H. Gan et al., "Pak2 kinase promotes cellular senescence and organismal aging," Proceedings of the National Academy of Sciences of the United States of America, vol. 116, no. 27, pp. 13311-13319, 2019.

[46] A. Strzeszewska, O. Alster, G. Mosieniak, A. Ciolko, and E. Sikora, "Insight into the role of PIKK family members and $\mathrm{NF}-\kappa \mathrm{B}$ in DNA damage-induced senescence and senescenceassociated secretory phenotype of colon cancer cells," Cell Death \& Disease, vol. 9, no. 2, p. 44, 2018.

[47] S. Takacova, R. Slany, J. Bartkova et al., "DNA damage response and inflammatory signaling limit the MLL-ENLinduced leukemogenesis in vivo," Cancer Cell, vol. 21, no. 4, pp. 517-531, 2012.

[48] V. Manfé, E. Biskup, P. Johansen et al., "MDM2 inhibitor nutlin-3a induces apoptosis and senescence in cutaneous T- 
cell lymphoma: role of p53," The Journal of Investigative Dermatology, vol. 132, no. 5, pp. 1487-1496, 2012.

[49] Y. Zhu, T. Tchkonia, T. Pirtskhalava et al., "The Achilles' heel of senescent cells: from transcriptome to senolytic drugs," Aging Cell, vol. 14, no. 4, pp. 644-658, 2015.

[50] J. Chang, Y. Wang, L. Shao et al., "Clearance of senescent cells by ABT263 rejuvenates aged hematopoietic stem cells in mice," Nature Medicine, vol. 22, no. 1, pp. 78-83, 2016.

[51] D. Mérino, S. L. Khaw, S. P. Glaser et al., "Bcl-2, Bcl-x(L), and $\mathrm{Bcl}-\mathrm{w}$ are not equivalent targets of $\mathrm{ABT}-737$ and navitoclax (ABT-263) in lymphoid and leukemic cells," Blood, vol. 119, no. 24, pp. 5807-5816, 2012.

[52] R. Bassan and D. Hoelzer, "Modern therapy of acute lymphoblastic leukemia," Journal of Clinical Oncology: Official Journal of the American Society of Clinical Oncology, vol. 29, no. 5, pp. 532-543, 2011.

[53] M. Ogura, T. Ishida, K. Tsukasaki, T. Takahashi, and A. Utsunomiya, "Effects of first-line chemotherapy on natural killer cells in adult T-cell leukemia-lymphoma and peripheral T-cell lymphoma," Cancer Chemotherapy and Pharmacology, vol. 78, no. 1, pp. 199-207, 2016.

[54] N. Mehta-Shah, L. Ratner, and S. M. Horwitz, “Adult T-cell leukemia/lymphoma," Journal of Oncology Practice, vol. 13, no. 8, pp. 487-492, 2017.

[55] O. A. O'Connor, G. Bhagat, K. Ganapathi et al., "Changing the paradigms of treatment in peripheral T-cell lymphoma: from biology to clinical practice," Clinical cancer research : an official journal of the American Association for Cancer Research, vol. 20, no. 20, pp. 5240-5254, 2014.

[56] I. Sturmlechner, M. Durik, C. J. Sieben, D. J. Baker, and J. M. van Deursen, "Cellular senescence in renal ageing and disease," Nature Reviews. Nephrology, vol. 13, no. 2, pp. 77-89, 2017.

[57] M. Ogrodnik, S. Miwa, T. Tchkonia et al., "Cellular senescence drives age-dependent hepatic steatosis," Nature Communications, vol. 8, no. 1, p. 15691, 2017.

[58] A. I. Robles, J. Jen, and C. C. Harris, "Clinical outcomes of TP53 mutations in cancers," Cold Spring Harbor Perspectives in Medicine, vol. 6, no. 9, 2016.

[59] A. Bisio, Y. Ciribilli, G. Fronza, A. Inga, and P. Monti, “TP53 mutants in the tower of babel of cancer progression," Human Mutation, vol. 35, no. 6, pp. 689-701, 2014.

[60] X. Yue, Y. Zhao, Y. Xu, M. Zheng, Z. Feng, and W. Hu, "Mutant p53 in cancer: accumulation, gain-of-function, and therapy," Journal of Molecular Biology, vol. 429, no. 11, pp. 1595-1606, 2017.

[61] H. Li, X. Yu, X. Liu et al., "Wogonoside induces depalmitoylation and translocation of PLSCR1 and N-RAS in primary acute myeloid leukaemia cells," Journal of Cellular and Molecular Medicine, vol. 22, no. 4, pp. 2117-2130, 2018.

[62] H. Li, H. Hui, J. Xu et al., "Wogonoside induces growth inhibition and cell cycle arrest via promoting the expression and binding activity of GATA-1 in chronic myelogenous leukemia cells," Archives of Toxicology, vol. 90, no. 6, pp. 1507-1522, 2016.

[63] X. Xu, X. Zhang, Y. Zhang et al., "Wogonin reversed resistant human myelogenous leukemia cells via inhibiting Nrf2 signaling by Stat3/NF- $\kappa \mathrm{B}$ inactivation," Scientific Reports, vol. 7, no. 1, p. 39950, 2017.

[64] D. Yang, Q. Guo, Y. Liang et al., "Wogonin induces cellular senescence in breast cancer via suppressing TXNRD2 expres- sion," Archives of Toxicology, vol. 94, no. 10, pp. 3433-3447, 2020.

[65] Y. Zhao, L. Zhang, Y. Wu et al., "Selective anti-tumor activity of wogonin targeting the Warburg effect through stablizing p53," Pharmacological Research, vol. 135, pp. 49-59, 2018.

[66] M. Mijit, V. Caracciolo, A. Melillo, F. Amicarelli, and A. Giordano, "Role of p53 in the regulation of cellular senescence," Biomolecules, vol. 10, no. 3, p. 420, 2020.

[67] A. J. Nakamura, Y. J. Chiang, K. S. Hathcock et al., "Both telomeric and non-telomeric DNA damage are determinants of mammalian cellular senescence," Epigenetics \& Chromatin, vol. 1, no. 1, p. 6, 2008.

[68] V. Gire, "Senescence: a telomeric limit to immortality or a cellular response to physiologic stresses?," Medecine sciences : M/S, vol. 21, no. 5, pp. 491-497, 2005.

[69] J. W. Shay, B. A. Van Der Haegen, Y. Ying, and W. E. Wright, "The frequency of immortalization of human fibroblasts and mammary epithelial cells transfected with SV40 large T-antigen," Experimental Cell Research, vol. 209, no. 1, pp. 45-52, 1993.

[70] E. Khattar and V. Tergaonkar, "Transcriptional regulation of telomerase reverse transcriptase (TERT) by MYC," Frontiers in cell and developmental biology, vol. 5, p. 1, 2017.

[71] M. V. Del Gaizo, J. R. Brown, M. Certo, T. M. Love, C. D. Novina, and A. Letai, "Chronic lymphocytic leukemia requires BCL2 to sequester prodeath BIM, explaining sensitivity to BCL2 antagonist ABT-737," The Journal of Clinical Investigation, vol. 117, no. 1, pp. 112-121, 2007.

[72] M. V. Del Gaizo, K. D. Schlis, S. E. Sallan, S. A. Armstrong, and A. Letai, "BCL-2 dependence and ABT-737 sensitivity in acute lymphoblastic leukemia," Blood, vol. 111, pp. 2300-2309, 2008.

[73] T. Oltersdorf, S. W. Elmore, A. R. Shoemaker et al., "An inhibitor of Bcl-2 family proteins induces regression of solid tumours," Nature, vol. 435, no. 7042, pp. 677-681, 2005. 